\title{
Assessment of BODIPY-Oxasmaragdyrin Dyads for Dye Sensitized Solar Cells: Aromaticity, Photosensitization Capability and Charge Transport
}

\author{
Merlys Borges-Martínez, ${ }^{\mathrm{a}}$ Daniel Alvarez, ${ }^{\mathrm{b}}$ Nicolas Montenegro-Pohlhammer, ${ }^{\mathrm{a}}$ M. Isabel \\ Menéndez, ${ }^{\mathrm{b}}$ Ramón López, ${ }^{\text {b }}$ Gloria Cárdenas-Jirón*a
}

${ }^{a}$ Laboratory of Theoretical Chemistry, Faculty of Chemistry and Biology, University of Santiago de Chile (USACH), 9170022, Santiago, Chile.

bDepartamento de Química Física y Analítica, Facultad de Química, Universidad de Oviedo, C/Julián Clavería 8, 33006 Oviedo, Asturias, Spain.

*Author to whom the correspondence should be addressed

*Email: gloria.cardenas@usach.cl

*Email: rlopez@uniovi.es 


\begin{abstract}
Dye-sensitized solar cells (DSSC) are presented as an alternative among renewable energies where the dye plays an important role to obtain an effective device. Our goal in this work is to examine the influence of several bridging functional groups between the BODIPY and oxasmaragdyrin systems forming dyads (D), as potential components of DSSC, on the aromatic, photophysical and charge transport properties of these ones. A set of 11 dyads made of the oxasmaragdyrin with 2,6dimethoxyphenyl and methylamine groups in two of their meso carbons (S) and the 4,4-difluoro-4bora-3a,4a-diaza-s-indacene (BODIPY, B) moieties that differ in the binding bridge between them has been analyzed with density functional theory (DFT). The geometries were optimized with the B3LYP/6-311G(d,p) level of theory employing D3 Grimme's correction, and a set of six functionals (B3LYP, BHandHLYP, CAM-B3LYP, PBE0, wB97X, TPSSh) were evaluated for reference systems in time dependent-DFT calculations. We found that TPSSh presents the best agreement with the available data of UV-vis spectra, so it was used for the calculation of the electronic absorption spectra of the 11 oxasmaragdyrins and 11 dyads. When the bridge between $\mathrm{S}$ and $\mathrm{B}$ consists of one (D3), two (D5), or three acetylene units (D6), a strong absorption band in the infrared region around $1000 \mathrm{~nm}$ can be achieved. These bands correspond to charge-separated excited states that favor a panchromatic absorption in that region. The aromaticity index $\operatorname{NICS}(0)$ computed at the macrocycle center ring critical point using the GIAO/B3LYP/6-311G(d,p) level of theory show in all these systems an aromatic character for the oxasmaragdyrin macrocycle (-10.7 to $-12.4 \mathrm{ppm})$. We also found that all dyads present a favorable electron injection toward the semiconductor $\mathrm{TiO}_{2}$ because the LUMO energy of the dyad is higher than the conduction band of the semiconductor $(-4.3 \mathrm{eV})$ used in a solar cell. Besides, the HOMO energy of the dyads D3, D5 and D6 is lower than the redox potential (-4.8 $\mathrm{eV}$ ) of a mediator as the $\mathrm{I}^{-} / \mathrm{I}_{3}{ }^{-}$system used to recover it after the circulation of electrons. Nonequilibrium Green`s function-based calculations performed for a couple of dyads; with (D6) and without (D4) a significant charge transfer band, connected to Au-electrodes show that D6 resulted to be a better conductor in agreement with the charge transfer results obtained from the photophysical properties. Finally, the Gibbs free energy for the formation of the dyads here investigated is calculated. All of them show to be exergonic reactions $\left(\Delta \mathrm{G}_{\text {solution }}<0\right)$ that suggests that these systems could be synthesized.
\end{abstract}




\section{INTRODUCTION}

The search for clean and renewable energy sources is a subject of great interest in our days because of the limited life of fossil fuels, the pollution caused by their combustion, and the growing environmental awareness. New technologies for the conversion of solar energy into electrical energy by means of dye-sensitized solar cells (DSSC) are a very attractive alternative due to their low cost and high performance. ${ }^{1-13}$ In these types of devices, the dye plays an important role whenever it meets the following requirements: a) radiation absorption in the visible-ultraviolet (UV-vis) and near-infrared (NIR) region, b) large molar absorption coefficients, c) energy of its lowest unoccupied molecular orbital (LUMO) above the conduction band edge of the semiconductor, with electrons located near the molecular group of the dye anchored to the semiconductor for an efficient electron injection, d) energy of its highest occupied molecular orbital (HOMO) below the redox mediator for an efficient electron reduction in the regeneration of the dye, and e) absence of aggregation on the semiconductor surface. ${ }^{14}$

Ruthenium complexes started being used as dyes in the DSSC since the pioneering work of Graztel et al. ${ }^{1-7}$ However, the limited availability, toxicity, and high cost of ruthenium have led an interest in looking for other efficient dyes for DSSC. Among them, porphyrins are attractive candidates because they have a rigid molecular structure of four pyrrolic rings connected by four bridging meso carbon atoms, with high absorption coefficients in the visible region and with many reaction centers (four meso positions and eight beta positions) suitable for the introduction of functional groups (see Scheme 1(a)). Several experimental ${ }^{15}$ 16 and theoretical ${ }^{17-20}$ studies have shown the viability of some porphyrins as dyes for DSSC. Expanded porphyrins containing more than four pyrrole rings, or alternative heterocycle subunits (see Scheme 1(b)) have emerged as functional organic chromophores for a wide range of applications because those structural modifications result in marked changes in the optical, redox, and ion-coordination properties. ${ }^{21-22}$ Due to their extended $\pi$-system, expanded porphyrins display red-shifted absorption bands and exceptionally large twophoton absorption cross-sections compared to the regular porphyrin. Consequently, they are excellent candidates for near-infrared dyes and nonlinear optical materials. Particularly, in 
recent years there has been growing research on expanded porphyrins consisting of five pyrrole rings in which a pyrrole unit is replaced by a furan named oxasmaragdyrin (see Scheme 1(c)).

(a)

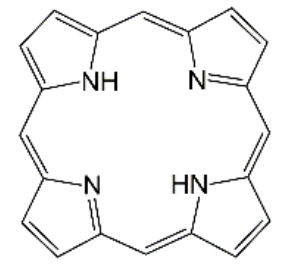

(b)

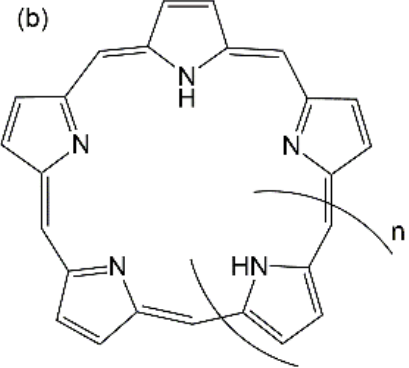

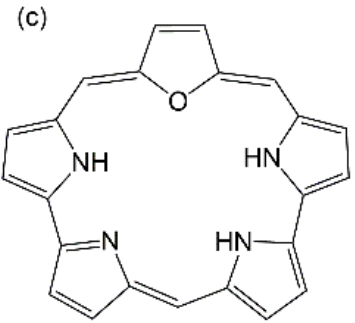

Scheme 1. Molecular structures of (a) porphyrin, (b) expanded porphyrin and (c) oxasmaragdyrin.

Recent studies have shown the importance of oxasmaragdyrin sensitizer as a compelling material for light-energy-harvesting applications. ${ }^{23-26}$ For instance, boryl oxasmaragdyrin offer many advantages, such as simpler synthesis and purification processes, low cost, and moisture stability. Besides, very recently, it has been found that such aromatic core-modified expanded porphyrins are efficient hole-transporting materials (HTMs) for perovskite solar cells (PSCs), as they retard the charge recombination and transport the photogenerated hole to the counter electrode. ${ }^{24}$

On the other hand, boron-dipyrromethene units (BODIPY) are also molecular systems commonly used for efficient light capture, mainly in the far visible or UV region of the electromagnetic spectrum. The photophysical properties of BODIPY and derivatives have been previously studied by Jacquemin`s and Russo`s groups. ${ }^{27-32}$ The coupling of oxasmaragdyrins with BODIPY, through different types of linkers, could also enhance the photosensitization properties of the porphyrin derivatives. ${ }^{23,33}$ Experimentally, the effect of spacers or bridges between chromophores has been studied. Among the bridges that have shown the best results in electronic transfer are those containing acene, ${ }^{34}$ thiophene ${ }^{35}$, and phenylethynyl $^{36}$ groups.

Recently, the synthesis of a covalently linked BODIPY-oxasmaragdyrin dyad has been reported $^{37}$ (see Scheme 2), where 4,4-difluoro-8-(4-ethynylphenyl)-4-bora-3a,4adiaza-s- 
indacene (BODIPY-CCH) reacts with a 5,10-(4-methylphenyl)-19-(4-iodophenyl)-25oxasmaragdyrin in the presence of toluene/triethylamine (3:1) and a catalytic mix of $\mathrm{Pd}_{2}(\mathrm{dba})_{3} / \mathrm{AsPh}_{3}$ at $45^{\circ} \mathrm{C}$ for $2 \mathrm{~h}$. The reaction has a yield of $65 \%$ for the formation of the dyad and is a stable product. The dyad presents absorption in the visible region in the range of $449-706 \mathrm{~nm}$.

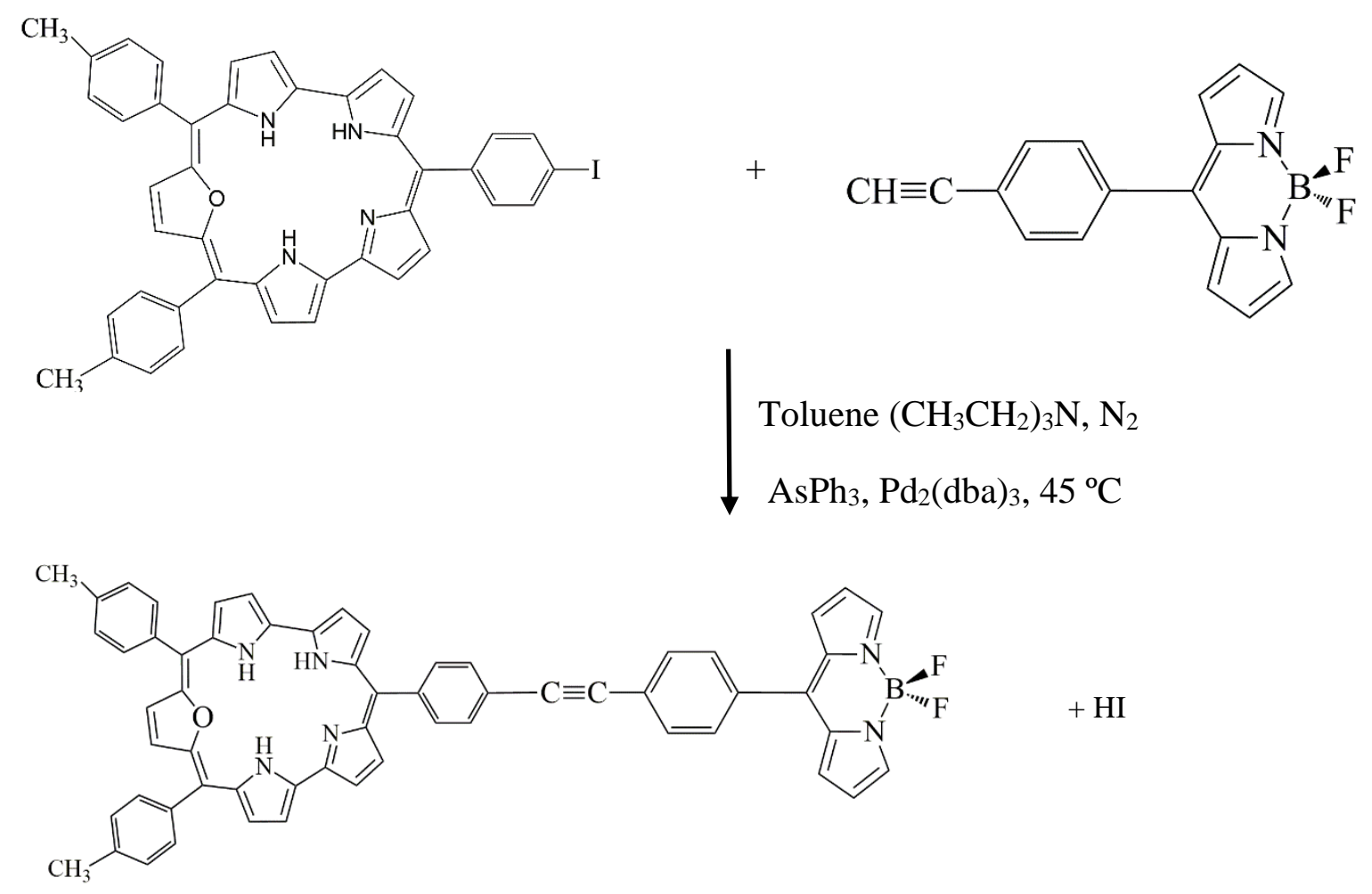

Scheme 2. The reaction for the synthesis of the BODIPY-oxasmaragdyrin dyad ${ }^{37}$.

Inspired by these authors and the characteristics of these compounds as photosensitizers, we have theoretically explored other linkers that could connect both chromophores, as also some structural variations for the dyad components; oxasmaragdyrin and BODIPY. To our knowledge, this kind of studies applied to BODIPY-oxasmaragdyrin dyads has not yet been reported in the literature. 


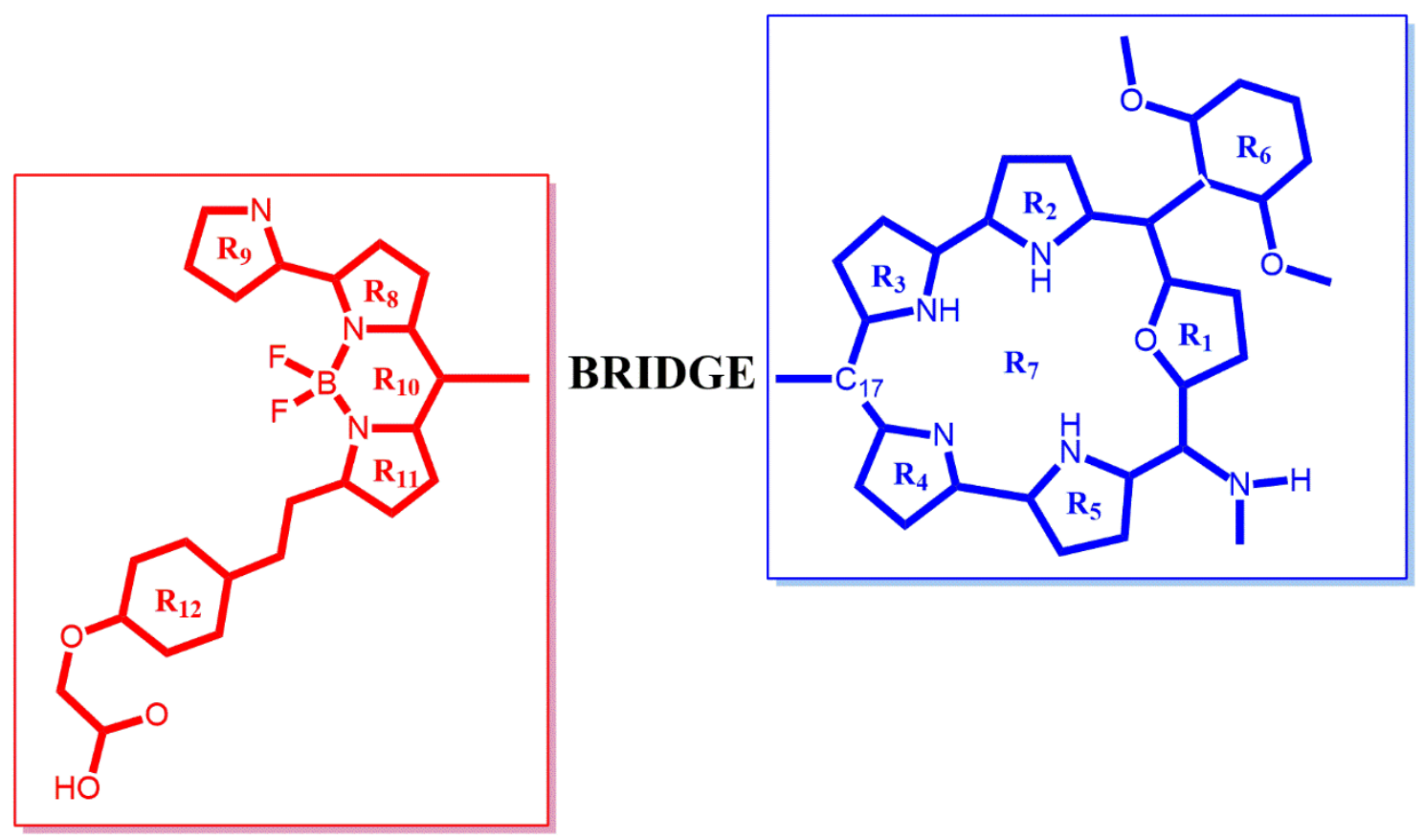

Notation

Dyad

D1

$\mathrm{B}-\mathrm{S}_{1}$

D2

$\mathrm{B}-\mathrm{CH}_{2}-\mathrm{CH}_{2}-\mathrm{CH}_{2}-\mathrm{S}_{1}$

D3

$\mathrm{B}-\mathrm{C} \equiv \mathrm{C}-\mathrm{S}_{1}$

D4

$\mathrm{B}-\mathrm{CH}_{2}-\mathrm{C} \equiv \mathrm{C}-\mathrm{S}_{1}$

D5

$\mathrm{B}-\mathrm{C} \equiv \mathrm{C}-\mathrm{C} \equiv \mathrm{C}-\mathrm{S}_{1}$

D6

$\mathrm{B}-\mathrm{C} \equiv \mathrm{C}-\mathrm{C} \equiv \mathrm{C}-\mathrm{C} \equiv \mathrm{C}-\mathrm{s}_{1}$

D7

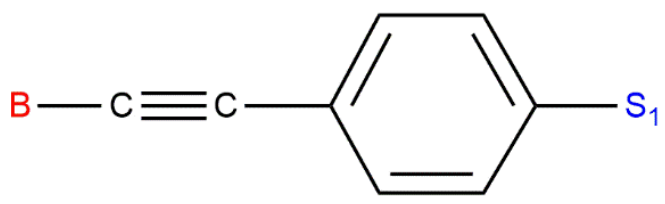

D8

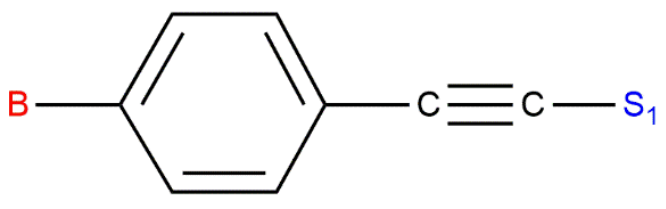


D9

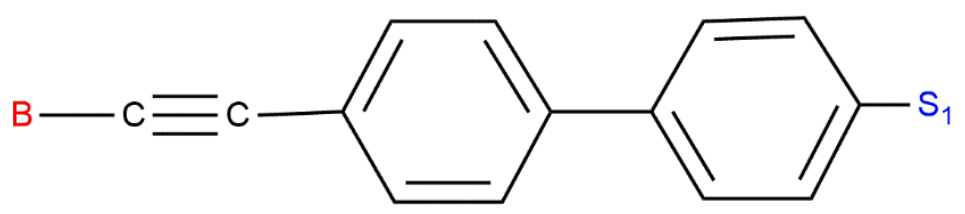

D10

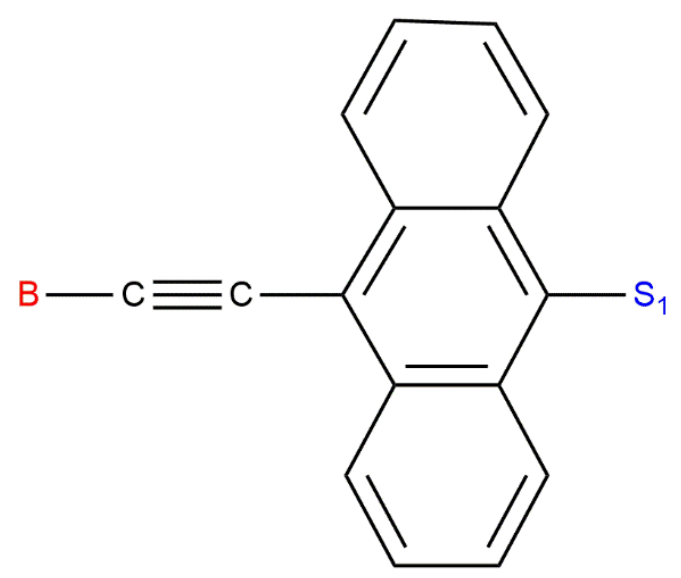

D11

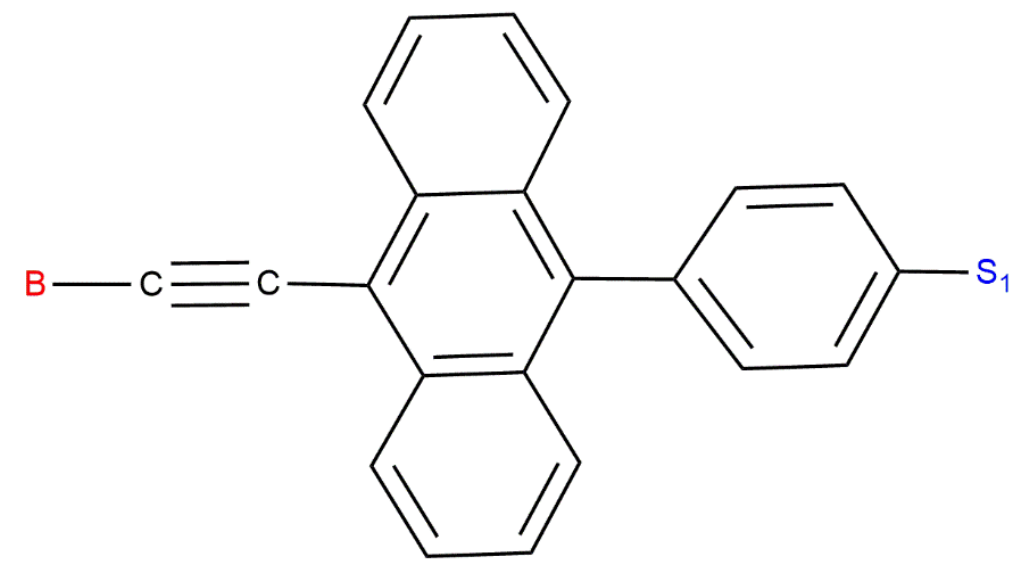

Scheme 3. Molecular structure of the dyads (D1-D11) formed by BODIPY (B) and the oxasmaragdyrin (S1) without and with different bridges.

The oxasmaragdyrin selected in this study has 2,6-dimethoxyphenyl and methylamine groups in two of its meso carbons (S1 in Scheme 3), which are electron-donating groups adequate to achieve the push-up effect of electron density from the porphyrin, ${ }^{15-16,38}$ towards the bridge, and eventually, to the BODIPY moiety. In addition, the selected BODIPY $(4,4-$ difluoro-4-bora-3a,4a-diaza-s-indacene, B in Scheme 3) is similar to a commercial BODIPY 650/660-succinimidyl ester, ${ }^{39}$ with a decrease in the size of its side chain. This modification ensures that electronic absorption properties are maintained while saving computational resources. 
The main goal of this theoretical research is to propose dyads composed of oxasmaragdyrin and BODIPY that could improve the photosensitization properties of the synthesized dyad displayed in Scheme 2. To that end, we examine the influence of several bridging functional groups between $\mathrm{B}$ and $\mathrm{S} 1$ on the photophysical properties of the BODIPY-oxasmaragdyrin dyads (see D2-D11 in Scheme 3). For the sake of a simple nomenclature, we include the bridge as part of the oxasmaragdyrin fragment in the dyad named S2-S11. Because of their structural characteristics, we also investigated the aromaticity of these systems and the binding nature between the fragments. A set of bridges between the two chromophores, including alkyl, phenyl, alkynyl groups and a combination of them was considered, evaluating their influence on the geometry, aromaticity and electronic absorption spectra. Finally, taking in consideration that some dyads present an important charge transfer between the fragments, we investigated the charge transport properties by simulating the dyad connected to gold electrodes to represent a molecular device.

\section{COMPUTATIONAL METHODS}

The optimization of the molecular structure of isolated oxasmaragdyrin S1 and its variants (S2-S11), the isolated BODIPY B, and the corresponding BODIPYoxasmaragdyrin dyads (D1-D11) was carried out applying the density functional theory (DFT) and the B3LYP ${ }^{40-42}$ functional as implemented in Gaussian 09 ${ }^{43}$ (see Scheme 3 for the investigated structures). B3LYP is a hybrid functional that combines the Becke's nonlocal exchange functional, the Hartree-Fock exchange energy, the nonlocal correlation functional of Lee, Yang, and Parr, and the Vosko-Wilk-Nusair fitting to the correlation energy derived from the local spin density approximation. All the atoms were described with the contracted triple- $\zeta$ quality basis set including polarization functions $6-311 \mathrm{G}(\mathrm{d}, \mathrm{p}),{ }^{44}$ while a modified version of the Schlegel algorithm ${ }^{45-47}$ was used to locate the energy minima on the potential energy surface. The nature of these critical points was confirmed by analytical computations of harmonic vibrational frequencies. The dispersion interactions in the B3LYP energy 
computations during the optimization were also taken into account through the calculation of the atom-pairwise DFT-D3 dispersion developed by Grimme and coworkers. $^{48}$

Aiming at finding out what factors can influence the sensitization properties of oxasmaragdyrin-type porphyrinic systems, we investigated the electronic absorption spectra and the aromaticity of the oxasmaragdyrins S1-S11 and their corresponding dyads D1-D11. On the one hand, B3LYP/6-311G(d,p) optimized geometries were used to calculate the excited electronic spectra applying the time-dependent DFT methodology (TD-DFT), ${ }^{49-50}$ where the electronic transitions are considered as verticals Franck-Condon type.

For regular porphyrins and some derivatives, the less expensive TD-DFT has been shown to be in better agreement with experiment than the more expensive ab initio configuration interaction (CI), complete active space second-order perturbation theory (CASPT2), or similarity transformed equation-of-motion coupled cluster (STEOMCC) calculations. ${ }^{51-52}$ To obtain reliable electronic spectra, we performed a calibration of some of the most commonly used density functionals in porphyrin-type systems: B3LYP, ${ }^{40-42}$ BHandHLYP, ${ }^{53}$ CAM-B3LYP, ${ }^{54}$ PBE0, ${ }^{55}$ TPSSh, ${ }^{56-57}$ and wB97x. ${ }^{58}$

To that end, we used as references the electronic absorption spectra experimentally reported for the BODIPY 650/660-succinimidyl ester ${ }^{39}\left(\mathrm{~B}_{\mathrm{ref}}\right)$ and oxasmaragdyrin 59 with phenyl substituents on the three meso carbons $\left(\mathrm{S}_{\mathrm{ref}}\right)$ (Scheme $\left.\mathrm{S} 1\right)$, which have very similar structures to those of the chromophores of our study. Particularly, we compared the electronic absorption spectra calculated with all the above-mentioned functionals for both the isolated BODIPY $\left(\mathrm{B}_{\mathrm{ref}}\right)$ and oxasmaragdyrin $\left(\mathrm{S}_{\mathrm{ref}}\right)$ with the corresponding reference spectra. Then, the best functional obtained was used to investigate the electron spectroscopic properties of S1-S11 and D1-D11. In these calculations, a set of 25,50, and 75 excited states were computed for oxasmaragdyrins (S1-S11), BODIPY, and dyads (D1-D11), respectively.

Solvent effects were taken into account in all the TD-DFT computations with the conductor-like polarizable continuum model (CPCM). ${ }^{60-61}$ The dielectric constants of $4.71,8.93$ and 32.61 were used to simulate the chloroform $\left(\mathrm{CHCl}_{3}\right)$, dichloromethane $\left(\mathrm{CH}_{2} \mathrm{Cl}_{2}\right)$ and methanol $\left(\mathrm{CH}_{3} \mathrm{OH}\right)$ solvents, respectively. $\mathrm{CH}_{2} \mathrm{Cl}_{2}$ is the solvent used in 
the TD-DFT computations on the S1-S11 and D1-D11 systems because the experimental absorption spectrum of $S_{\text {ref }}$ was obtained in that solvent ${ }^{59}$ and the absorption spectrum of BODIPY is relatively insensitive to the solvent. ${ }^{39} \mathrm{CHCl}_{3}$ and $\mathrm{CH}_{3} \mathrm{OH}$ were only used in the TD-DFT calculations on the dyad shown in Scheme 2 and the isolated BODIPY B to compare them with the electron absorption spectrum reported for $\mathrm{D}_{\text {ref }}$ and $\mathrm{B}_{\text {ref }}$ in such solvents, respectively. ${ }^{52}$ All the relevant molecular orbitals involved in the electronic transitions of each system were computed.

On the other hand, the aromaticity of all the structures located was investigated in terms of the magnetic criterion based on NICS (nucleus-independent chemical shift) calculations $^{62-63}$ using the Gauge-including atomic orbital (GIAO) method in conjunction with the B3LYP/6-311G(d,p) level of theory. NICS values were computed at the ring critical point (RCP) located inside the oxasmaragdyrin macrocycle, $\operatorname{NICS}(0)$. Some theoretical studies on related systems recommend to compute the NICS not at the geometric center but at the point of lowest density in the ring plane, i.e., at the RCP. ${ }^{64}$ This recommendation has proven to be particularly appropriate in the case of heterocyclic or metalloaromatic compounds, where the RCP is usually displaced from the geometric ring center. Here, RCPs were detected by making a topological analysis of the electron density based on the Atoms-inMolecules theory of Bader (AIM) ${ }^{65-67}$ using the AIMAll program. ${ }^{68}$ Although other NICS definitions like that computed at $1 \AA$ above RCP, NICS(1), and its out-of-plane tensor component, NICS(1)zz, were recommended as a better measure of the $\pi$ electron delocalization compared to $\operatorname{NICS}(0),{ }^{69-70}$ the former indices are not adequate to measure the macrocycle aromaticity of twisted-Hückel conformations of expanded porphyrins. $^{71}$ Therefore, in the present work, we only consider NICS(0) values. Magnetically induced current densities were also calculated at the B3LYP/6$311 G(d, p)$ level using the AIMAll program. ${ }^{72-73}$ The analyses of calculated current densities and current pathways have proved to be useful for obtaining a more detailed picture of the aromatic pathways in porphyrin systems. ${ }^{74-76}$ A better understanding of the aromatic properties may help in designing porphyrinoids with selected properties.

In order to evaluate the electronic transport properties of the dyads, the molecular junction systems composed of each of the aforementioned structures were anchored 
to two $\mathrm{Au}(111)$ nanowires by means of thiol groups. These properties were calculated using the Nanodcal program suite ${ }^{77}$, which is based on a combination of DFT with the Keldysh nonequilibrium Green's function (NEGF) formalism. ${ }^{78}$ To this end, the molecular structure of each dyad with two of their terminal hydrogen atoms substituted for S-Au groups, were optimized at the DFT level of theory (BP86 ${ }^{40}$, 79 /def2-SVP ${ }^{80-81}$ ) with the D3 Grimme`s dispersion correction. To represent the gold atoms, the relativistic Stuttgart pseudopotentials were employed. Then these atoms were attached to the hollow site of the $\mathrm{Au}$ (111) electrode extensions.

For the calculation of the transport properties, all the atoms were represented by a double-zeta polarized (DZP ${ }^{82-83}$ ) basis set, using the PBE96 ${ }^{84}$ exchange-correlation functional. The mesh cut-off energy was set to 150 Rydberg for both the real and reciprocal space grids in all calculations, with a self-consistency tolerance for the convergence of the Hamiltonian and density matrices of $3.6 \times 10^{-6}$ a.u.

\section{RESULTS AND DISCUSSION}

3.1 Molecular Structures. In this section, the planarity of the oxasmaragdyrin is analyzed, first as an isolated fragment, then when linked to the different bridges, and finally when those bridges connect the porphyrin derivative with the BODIPY moiety.

From the structural point of view, oxasmaragdyrin (S1) is an expanded porphyrin made of five rings and three meso carbon atoms. As an isolated fragment, it strongly deviates from planarity with a root mean square deviation (RMSD) of $0.31 \AA$ with respect to the best plane defined by the atoms of the core of the porphyrin that involves the pyrrole rings (see Scheme 3). $\beta$ carbon atoms of R2 and R5 rings stand below the reference plane, yielding a dome geometry for the macrocycle. When bridges are connected to C17 (S2-S11), the planarity of the oxasmaragdyrin macrocycle increases, regardless of the nature of the bridge, as it is reflected in the lower RMSD values collected in Table 1 for S2-S11. These values go from 0.12 (S3, S4, S6) to $0.05 \AA$ (S9). Bridges in S7, S9, S10, and S11 provide a phenyl derivative ring directly linked to $\mathrm{S} 1$ and render large planarity of the macrocycle. As an illustration, Figure S1 shows a molecular view of the oxasmaragdyrins with the highest (S1) and lowest (S9) RMSD values. The joining of the BODIPY moiety to the corresponding oxasmaragdyrin 
hardly affects the planarity of the porphyrin derivative ( $\triangle \mathrm{RMSD}$ is always lower than 0.08 $\AA$, as seen in the last column of Table 1). The only exception is D1, since in this case, the unsubstituted non-planar S1 becomes a C17 substituted oxasmaragdyrin with a degree of planarity very similar to most of the other dyads (its RMSD reduces in $0.21 \AA$ ).

Structurally speaking, it is interesting to mention the relative orientation of both end moieties in the dyads (see Figure S2). In the absence of a bridge, as in D1, the oxasmaragdyrin macrocycle and the BODIPY core lie in almost perpendicular planes. Thus, steric repulsions between neighbor $\mathrm{H}$ atoms in the binding region are minimized. Nearly perpendicular orientation of the planes containing both terminal cycles is also found for D2, D4, D7, D8, and D10. In the remaining dyads both units are almost coplanar, although as the length of the bridge increases, their relative orientation depends more on the nature of the bridge than on their own interactions.

Table 1. Root-mean square deviation (RMSD, $\AA$ ) of the non-hydrogen atoms of the oxasmaragdyrin core to the best plane fitting the set of Cartesian coordinates of such atoms by a least squares method for the different oxasmaragdyrins and their respective dyads investigated at the B3LYP/6-311G(d,p) level and the difference between them ( $\triangle \mathrm{RMSD}, \AA$ ).

\begin{tabular}{ccccc}
\hline Oxasmaragdyrin & RMSD & Dyad & RMSD & $\Delta$ RMSD \\
\hline S1 & 0.31 & D1 & 0.11 & -0.21 \\
S2 & 0.09 & D2 & 0.15 & 0.06 \\
S3 & 0.12 & D3 & 0.14 & 0.03 \\
S4 & 0.12 & D4 & 0.13 & 0.01 \\
S5 & 0.13 & D5 & 0.11 & -0.02 \\
S6 & 0.12 & D6 & 0.11 & -0.01 \\
S7 & 0.08 & D7 & 0.11 & 0.03 \\
S8 & 0.11 & D8 & 0.14 & 0.03 \\
S9 & 0.05 & D9 & 0.13 & 0.08 \\
S10 & 0.06 & D10 & 0.13 & 0.06 \\
S11 & 0.11 & D11 & 0.11 & 0.00 \\
\hline
\end{tabular}

\subsection{Spectroscopic Properties}


Calibration of the Density Functional. To obtain theoretically the electronic absorption spectra of the dyads and to analyze their corresponding excited states, a calibration method looking for the density functional that best reproduces the experimental data was previously performed for the selected fragments $\left(\mathrm{S}_{\mathrm{ref}}, \mathrm{B}_{\mathrm{ref}}\right)$, where the UV-vis spectra are available. Tables 2 and 3 show the characteristic bands calculated for these fragments using the B3LYP, BHandHLYP, CAM-B3LYP, PBE0, wB97X, and TPSSh functionals, along with the measured values. ${ }^{39,59} \mathrm{We}$ include the wavelength $(\mathrm{nm})$, excitation energy $(\mathrm{eV})$, the oscillator strength, and the deviation of the theoretical values with respect to the experimental ones (in $\mathrm{eV}$ and \%).

Table 2. Excitation wavelength $(\lambda)$ and energy (E), oscillator strength $(f)$ and assignment of the main absorption bands of oxasmaragdyrin $\left(S_{\text {ref }}\right)$ calculated in $\mathrm{CH}_{2} \mathrm{Cl}_{2}$ and using the 6-311G(d,p) basis set. Experimental deviation ${ }^{59}$ in square parenthesis $(\mathrm{eV}, \%)$.

\begin{tabular}{ccc}
\hline Density Functional & $\lambda / \mathrm{nm}(\mathrm{E} / \mathrm{eV}) f$ & Band \\
\hline B3LYP & $627(1.98) 0.137[0.02,1 \%]$ & $\mathrm{Q}$ \\
& $600(2.07) 0.025[\mathbf{0 . 0 3}, \mathbf{2 \%}]$ & $\mathrm{Q}$ \\
& $446(2.78) 1.890[0.02,1 \%]$ & Soret \\
BHandHLYP & $589(2.10) 0.022[0.15,7 \%]$ & $\mathrm{Q}$ \\
& $577(2.15) 0.033[0.05,2 \%]$ & $\mathrm{Q}$ \\
CAM-B3LYP & $403(3.08) 2.290[\mathbf{0 . 2 8 , 9 \%}$, & Soret \\
& $599(2.07) 0.030[0.11,5 \%]$ & $\mathrm{Q}$ \\
PBE0 & $580(2.14) 0.152[0.04,2 \%]$ & $\mathrm{Q}$ \\
& $404(3.07) 2.130[\mathbf{0 . 2 7}, \mathbf{9 \%}]$ & Soret \\
& $613(2.02) 0.143[\mathbf{0 . 0 6}, \mathbf{3 \%}]$ & $\mathrm{Q}$ \\
wB97X & $589(2.10) 0.026[0.01,0,3 \%]$ & $\mathrm{Q}$ \\
& $436(2.84) 1.990[0.04,2 \%]$ & Soret \\
& $613(2.02) 0.016[0.06,3 \%]$ & $\mathrm{Q}$ \\
TPSSh & $521(2.38) 0.162[0.28,12 \%]$ & $\mathrm{Q}$ \\
& $375(3.31) 2.170[\mathbf{0 . 5 1 , 1 5 \%}]$ & Soret \\
& $637(1.95) 0.140[0.01,1 \%]$ & $\mathrm{Q}$ \\
& $605(2.05) 0.025[0.05,2 \%]$ & $\mathrm{Q}$ \\
EXP & $455(2.72) 1.820[\mathbf{0 . 0 7}, \mathbf{3 \%}]$ & Soret \\
& $696(1.78), \mathbf{6 3 3}(1.96)$ & $\mathrm{Q}$ \\
& $\mathbf{5 9 1}(2.10), 552(2.25)$ & $\mathrm{Q}$ \\
& $\mathbf{4 4 3 ( 2 . 8 0 )}$ & Soret \\
\hline
\end{tabular}

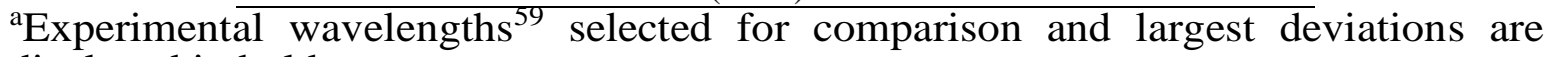
displayed in bold. 
For oxasmaragdyrin $\left(\mathrm{S}_{\mathrm{ref}}\right)$, we note that all the functionals predict bands in the visible region, two weak $\mathrm{Q}$ bands, and one strong Soret band, as expected for an expanded porphyrin. Obtaining in the calculations two $\mathrm{Q}$ bands instead of four occurs because the bands given by TD-DFT are not pure states, but a combination of electronic transitions. The $\mathrm{Q}$ band at the longest wavelength corresponds in all the functionals to $\mathrm{H}-1 \rightarrow \mathrm{L}+1$ and $\mathrm{H} \rightarrow \mathrm{L}$ transitions of the $\mathrm{Q}(1-1)$ and $\mathrm{Q}(0-0)$ types, respectively, while the $\mathrm{Q}$ band at the lowest wavelength corresponds to $\mathrm{H}-1 \rightarrow \mathrm{L}$ and $\mathrm{H} \rightarrow \mathrm{L}+1$ transitions of the type Q (1-0) and Q (0-1) types, respectively.

Table 3. Excitation wavelength $(\lambda)$ and energy (E), oscillator strength $(f)$ and assignment of the main absorption bands of BODIPY $\left(\mathrm{B}_{\text {ref }}\right)$ calculated in $\mathrm{CH}_{3} \mathrm{OH}$ and using the $6-311 \mathrm{G}(\mathrm{d}, \mathrm{p})$ basis set. Experimental deviation ${ }^{39}$ in square parenthesis $(\mathrm{eV}$, $\%)$.

\begin{tabular}{clc}
\hline $\begin{array}{c}\text { Density } \\
\text { Functional }\end{array}$ & \multicolumn{1}{c}{$\lambda / \mathrm{nm}(\mathrm{E} / \mathrm{eV}) f$} & Assignment \\
\hline B3LYP & $621(2.00) 0.854[0.08,4 \%]$ & 1 \\
& $339(3.66) 0.399[0.13,3 \%]$ & 2 \\
& $217(5.72) 0.319[\mathbf{1 . 4 1}, \mathbf{2 5 \%}]$ & 3 \\
BHandHLYP & $583(2.13) 0.906[\mathbf{0 . 2 1}, \mathbf{1 0 \%}]$ & 1 \\
& $352(3.52) 0.696[0.01,0.4 \%]$ & 2 \\
& $296(4.19) 0.588[0.11,3 \%]$ & 3 \\
CAM-B3LYP & $589(2.11) 0.872[\mathbf{0 . 1 9}, \mathbf{9 \%}]$ & 1 \\
& $353(3.52) 0.744[0.02,0.5 \%]$ & 2 \\
PBE0 & $296(4.19) 0.639[0.12,3 \%]$ & 3 \\
& $610(2.03) 0.896[0.11,6 \%]$ & 1 \\
& $331(3.74) 0.564[0.21,6 \%]$ & 2 \\
wB97X & $212(5.84) 0.371[\mathbf{1 . 5 3}, \mathbf{2 6 \%}]$ & 3 \\
& $578(2.14) 0.877[0.22,10 \%]$ & 1 \\
& $323(3.83) 1.134[\mathbf{0 . 3 0 , 8 \%}]$ & 2 \\
TPSSh & $275(4.51) 0.373[0.21,5 \%]$ & 3 \\
& $628(1.98) 0.855[0.06,3 \%]$ & 1 \\
& $353(3.51) 0.380[0.02,1 \%]$ & 2 \\
EXP $^{\mathrm{a}}$ & $293(4.24) 0.289[\mathbf{0 . 0 7}, \mathbf{2 \%}]$ & 3 \\
& $646(1.92) 100^{\mathrm{b}}$ & 1 \\
& $351(3.53) 35.7$ & 2 \\
\hline
\end{tabular}


${ }^{\text {a}}$ Experimental wavelengths selected for comparison and largest deviation are

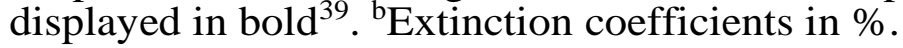

The theoretical bands for $S_{\text {ref }}$ are compared to the Q bands measured at 633 and 591 $\mathrm{nm}$, and to Soret band at $443 \mathrm{~nm} .^{59}$ The lowest deviations for both types of bands are found for B3LYP (0.03 eV), PBE0 $(0.06 \mathrm{eV})$, and TPSSh $(0.07 \mathrm{eV})$, which for the TD-DFT methodology are considered as negligible errors. These three functionals share the characteristic that they are hybrid functionals with a similar contribution of Hartree Fock (HF) exact electronic exchange energy, 20\%, 28\%, and 13\%, respectively. Range-separated functionals like CAM-B3LYP and wB97X predict larger errors of up to 0.27 and $0.51 \mathrm{eV}$, respectively, which may be attributed to the larger contribution of $\mathrm{HF}$ exchange for long-range terms of $65 \%$ and $100 \%$, respectively. BHandHLYP with $50 \%$ HF exchange generates a deviation of up to 0.28 $\mathrm{eV}$, similar to CAM-B3LYP, although somewhat smaller than that of wB97X, represents an important error for TD-DFT calculations. Based on the results, it is clear that functionals with a percentage of $\mathrm{HF}$ exchange greater than $28 \%$ do not describe well the absorption bands of oxasmaragdyrin $\mathrm{S}_{\text {ref. }}$ It is a macrocycle with a strongly covalent electronic structure with high delocalization of $\pi$-electrons. Non-bonding interactions can occur between the lone pairs of oxygen atoms and the closest inner hydrogen atoms of the pyrrole rings. However, they do not represent interactions of large magnitude.

In the case of BODIPY ( $\mathrm{B}_{\text {ref }}$ ), all the functionals predict three bands as experimentally observed $(646,351$ and $288 \mathrm{~nm})$, one of them in the visible region and the other two in the UV region. The deviations obtained with the hybrid functionals are much higher than those obtained for $S_{\text {ref, }} 1.41$ and $1.53 \mathrm{eV}$ for B3LYP and PBE0, respectively. The TPSSh functional predicts the same small error $(0.07 \mathrm{eV})$ as for $\mathrm{S}_{\text {ref. }}$ The range-separated functionals present a better behavior than for $S_{\text {ref, with }} 0.19$ and $0.30 \mathrm{eV}$ deviations for CAM-B3LYP and wB97X, respectively. BHandHLYP predicts similar values to CAM-B3LYP $(0.21 \mathrm{eV})$. In the case of BODIPY, the range-separated functionals lead to a better description of the excited states than for $S_{\text {ref. }}$ It could be attributed to the $\mathrm{BF}_{2}$ group, where the fluorine atoms present non-bonding interactions as hydrogen bonding with the closer hydrogen atoms. 
Taking into consideration all that was mentioned above, TPSSh is the functional showing the best agreement with the experimental data of both oxasmaragdyrin $S_{\text {ref }}$ and BODIPY $B_{\text {ref. }}$

The same functionals were also tested for the reference dyad ( $\left.\mathrm{D}_{\mathrm{ref}}\right)$ (Schemes 2 and S1(c)) whose molecular structure is very similar to D7 and D8 of our work (Scheme 3). The molecular structure of $D_{\text {ref }}$ was also optimized at the B3LYP/6-311G(d,p) level. We found that all the functionals show important errors, except TPSSh (0.01 $\mathrm{eV}$ ), especially for the BODIPY band with deviations in the range of $0.42-0.50 \mathrm{eV}$. A comparison between the theoretical (TPSSh) and experimental electronic absorption spectra for $\mathrm{D}_{\text {ref }}$ is shown in Figure 1 (see also Table S1). As can be seen, there is an excellent agreement between the experimental and theoretical results for this system. In the 550-710 $\mathrm{nm}$ region, four small bands assigned as $\mathrm{Q}$ bands were observed experimentally. TPSSh functional predicts two of them with a deviation of $0.01 \mathrm{eV}$. In addition, both Soret and BODIPY bands are well predicted with deviations of 0.05 and $0.01 \mathrm{eV}$, respectively.

In summary, we have demonstrated that TPSSh functional predicts adequately the optical properties of this kind of molecular systems, therefore we choose it for making the study of the electronic absorption properties of the new dyads.

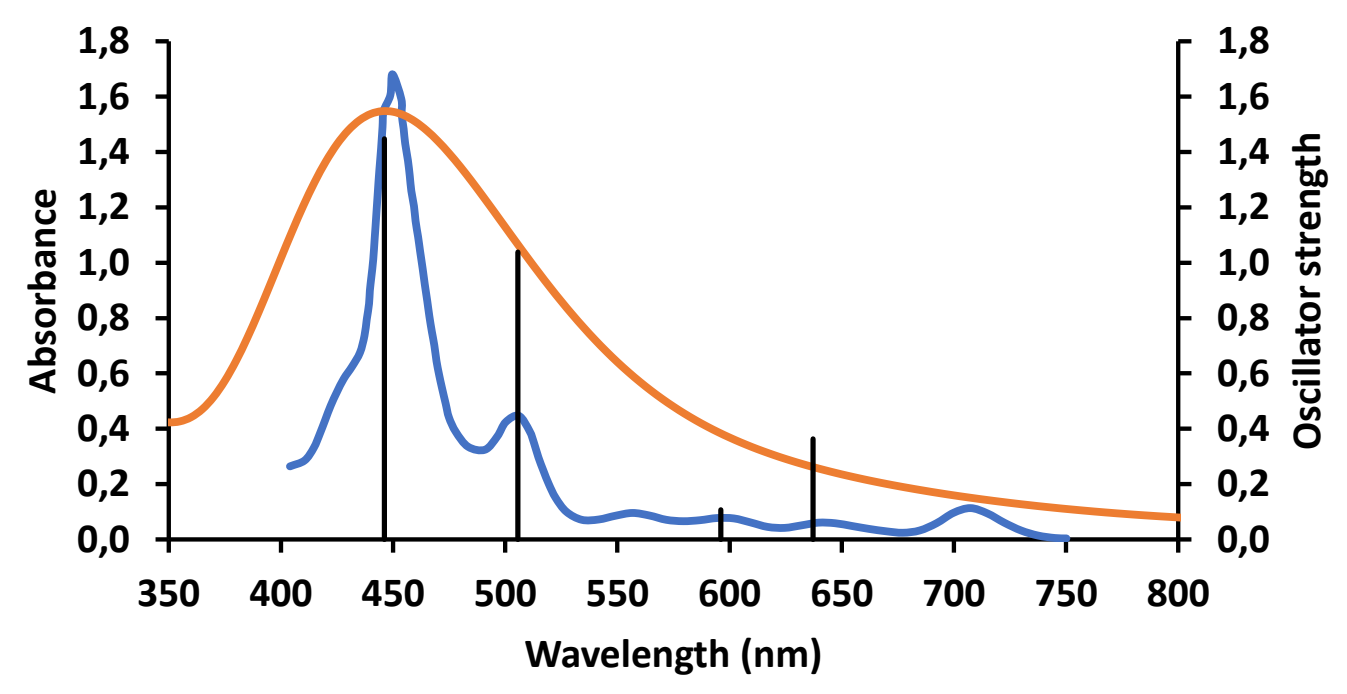


Figure 1. Comparison between the experimental (blue lines) and theoretical (TPSSh, orange line) electronic absorption spectrum of $\mathrm{D}_{\text {ref. }}$ Black lines correspond to the theoretical absorption values for the Soret, Q and BODIPY bands.

Photophysical Properties. Calculated photophysical properties for the oxasmaragdyrins S1-S11 and their corresponding dyads D1-D11 shown in Scheme 3 and calculated at the TPSSh/6-311G(d,p) level of theory in $\mathrm{CH}_{2} \mathrm{Cl}_{2}$ are presented in Table 4. Excitation wavelength and energy for each characteristic band of both oxasmaragdyrin and BODIPY fragments are included in that table. Details about the oscillator strength and the electronic transition associated with each band of the dyads are shown in Table S2.

All these compounds retain the characteristic Q and Soret absorption bands of the reference oxasmaragdyrin $S_{\text {ref }}$ as well as the typical visible absorption band of the reference BODIPY $B_{\text {ref }}$ in the case of the dyad systems. Let us now discuss the effect of bridging groups on these bands, first for S2-S11, and then for D1-D11.

Concerning the comparison of the photophysical properties of S2-S11 with those of S1, all the bridges cause an increase of the wavelengths of the Q and Soret bands ( $\lambda_{\mathrm{Q}}$ and $\lambda_{\text {Soret, }}$, respectively) except for $\mathrm{S} 2$. That is, the linkage of the alkyl group $\mathrm{CH}_{3-}$ $\mathrm{CH}_{2}-\mathrm{CH}_{2}$ - to $\mathrm{S} 1$ decreases the two $\lambda_{\mathrm{Q}}$ by 21 and $5 \mathrm{~nm}$, respectively, and $\lambda_{\text {Soret }}$ by 6 nm. At S3, with one acetylene (Ace) group bonded to S1, all the absorption bands are red-shifted by 24 and $22 \mathrm{~nm}$ (Q bands), and $15 \mathrm{~nm}$ (Soret band). The addition of a methyl group at the end of the chain, as in S4, hardly affects the position of the absorption bands with respect to S3. However, the addition of either more acetylene units, as in S5 and S6, or a phenyl group, as in S8, increases the $\lambda_{\mathrm{Q}}$ bands between 54 and $83 \mathrm{~nm}$ and $\lambda_{\text {Soret }}$ by more than $20 \mathrm{~nm}$, which indicates the existence of conjugation between S1 and linkers with an acetylene moiety directly bonded to it.

When a phenyl group is directly attached to $S 1$, as in $S 7$, the two $\lambda_{Q}$ values hardly increase, while an increase of $21 \mathrm{~nm}$ was found for the $\lambda_{\text {Soret }}$ value. Similarly, one biphenyl group replacing the phenyl, as in $S 9$, renders $\lambda_{\mathrm{Q}}$ and $\lambda_{\text {Soret }}$ values only slightly greater than those of S1. The small effect of the bridges with phenyl and biphenyl 
connections to $\mathrm{S} 1$ comes from the absence of co-planarity between these rings and $\mathrm{S} 1$, thus hindering the electron delocalization. Surprisingly, S10 and S11, with an anthracene group in the chain and no possible expansion of the electron density from the oxasmaragdyrin to the nearly perpendicular bridge rings, show $\lambda_{\mathrm{Q}}$ and $\lambda_{\text {Soret }}$ values much larger than those of S1, mainly for S10, where the anthracene directly binds to S1.

Table S2 collects detailed information about the calculated absorption spectra, showing the molecular orbitals (MOs) involved in the transitions. For S9, and mainly $\mathrm{S} 10$ and $\mathrm{S} 11$, the MO LUMO+2 $(\mathrm{L}+2)$ receives electrons in $\mathrm{Q}$ and Soret excitations. This orbital is essentially placed in the biphenyl or anthracene moieties, which means that new and low energy (long wavelength) oxasmaragdyrin $\rightarrow$ bridge transitions are present.

Let us see now the effect that the bonding of the BODIPY unit to S1-S11 produces on the oxasmaragdyrin absorption bands just described.

For the Soret band, the anchorage of B to S1-S11 slightly increases all the $\lambda_{\text {Soret }}$ values in the 0-7 nm range, except for the Ace-phenyl spacer (D7), which causes a decrease of $10 \mathrm{~nm}$ when going from S7 to D7. For the Q bands, some dyads reduce their $\lambda_{\mathrm{Q}}$ values with respect to those in the corresponding oxasmaragdyrin, and others hardly modify them. In the first group, D3, D5, and D6 allow certain B-bridge conjugation that, consequently, reduces the bridge-S one, which explains the reduction in the oxasmaragdyrin $\lambda_{\mathrm{Q}}$ bands. Besides, D10 and mainly D11, also reduce the wavelength of these bands but now it is due to the presence of large energy transitions starting in the MO HOMO-3 (H-3) located at the anthracene moiety. On the other hand, $\lambda_{\mathrm{Q}}$ values of D1, D2, D4, D7, D8, and D9 keep the value they had in the corresponding oxasmaragdyrins, due to the isolation of both fragments produced by the nearly perpendicular conformation of close rings (D1, D7, D8, and D9) or by the presence of an alkyl fragment linked with B (D2 and D4). The visible band of B is also affected by the presence of S1-S11. Thus, Table 4 shows that, compared to D1, D2-D6 reduce its value, while D7-D11 increase it, making the band of B the one with the largest value. Looking for panchromatism, a large absorption wavelength range is needed, that is, the Soret band, which has the shortest wavelength, should be shifted 
to even lower values, and the band corresponding to the largest wavelength to larger values. Dyads do not significantly reduce the value of the Soret band, while D7-D11 increase the B visible band as just said. Among this group, D7 (between 448 and 707 $\mathrm{nm}$ ) and D9 (between 448 and $705 \mathrm{~nm}$ ) have the widest absorption range, shifting the gap of isolated oxasmaragdyrin S1 $(437-636 \mathrm{~nm})$ towards the IR region.

Table 4. Excitation wavelength $(\lambda)$ and energy (E), and assignment of the main absorption bands of the oxasmaragdyrins S1-S11 and the corresponding dyads D1D11 calculated at the TPSSh/6-311G(d,p) level of theory in the solution phase $\left(\mathrm{CH}_{2} \mathrm{Cl}_{2}\right)$.

\begin{tabular}{cccccc}
\hline & $\lambda / \mathrm{nm}(\mathrm{E} / \mathrm{eV})$ & Band & & $\lambda / \mathrm{nm}(\mathrm{E} / \mathrm{eV})$ & Band \\
\hline S1 & & & $\mathrm{D} 1$ & $640(1.94)$ & BODIPY \\
& $636(1.95)$ & $\mathrm{Q}$ & & $635(1.95)$ & $\mathrm{Q}$ \\
& $588(2.11)$ & $\mathrm{Q}$ & & $599(2.07)$ & $\mathrm{Q}$ \\
& $437(2.84)$ & Soret & & $438(2.83)$ & Soret \\
\hline S2 & & & $\mathrm{D} 2$ & $629(1.97)$ & BODIPY \\
& $615(2.02)$ & $\mathrm{Q}$ & & $626(1.98)$ & $\mathrm{Q}$ \\
& $583(2.13)$ & $\mathrm{Q}$ & & $587(2.11)$ & $\mathrm{Q}$ \\
& $431(2.88)$ & Soret & & $437(2.83)$ & Soret \\
\hline S3 & & & $\mathrm{D} 3$ & $555(2.23)$ & BODIPY \\
& $660(1.88)$ & $\mathrm{Q}$ & & $622(1.99)$ & $\mathrm{Q}$ \\
& $610(2.03)$ & $\mathrm{Q}$ & & $582(2.13)$ & $\mathrm{Q}$ \\
& $452(2.74)$ & Soret & & $459(2.70)$ & Soret \\
\hline S4 & & & $\mathrm{D} 4$ & $639(1.94)$ & BODIPY \\
& $653(1.90)$ & $\mathrm{Q}$ & & $662(1.87)$ & $\mathrm{Q}$ \\
& $608(2.04)$ & $\mathrm{Q}$ & & $610(2.03)$ & $\mathrm{Q}$ \\
& $452(2.74)$ & Soret & & $453(2.74)$ & Soret \\
\hline S5 & & & D5 & $569(2.18)$ & BODIPY \\
& $690(1.80)$ & $\mathrm{Q}$ & & $656(1.89)$ & $\mathrm{Q}$ \\
& $629(1.97)$ & $\mathrm{Q}$ & & $581(2.13)$ & $\mathrm{Q}$ \\
& $459(2.70)$ & Soret & & $459(2.70)$ & Soret \\
\hline S6 & & & D6 & $585(2.12)$ & BODIPY \\
& $719(1.72)$ & $\mathrm{Q}$ & & $688(1.80)$ & $\mathrm{Q}$ \\
& $650(1.91)$ & $\mathrm{Q}$ & & $605(2.05)$ & $\mathrm{Q}$ \\
& $472(2.63)$ & Soret & & $479(2.59)$ & Soret \\
\hline S7 & & & D7 & $707(1.75)$ & BODIPY \\
& $643(1.93)$ & Q & & $642(1.93)$ & Q \\
& $596(2.08)$ & Q & & $594(2.09)$ & Q \\
& $458(2.71)$ & Soret & & $448(2.77)$ & Soret \\
\hline S8 & & & D8 & $667(1.86)$ & BODIPY \\
& $691(1.79)$ & Q & & $689(1.80)$ & Q
\end{tabular}




\begin{tabular}{cccccc} 
& $628(1.97)$ & Q & & $624(1.99)$ & Q \\
& $470(2.64)$ & Soret & & $471(2.63)$ & Soret \\
\hline S9 & & & D9 & $705(1.76)$ & BODIPY \\
& $640(1.94)$ & Q & & $645(1.92)$ & Q \\
& $595(2.08)$ & Q & & $596(2.08)$ & Q \\
& $442(2.80)$ & Soret & & $448(2.77)$ & Soret \\
\hline S10 & & & D10 & $697(1.78)$ & BODIPY \\
& $687(1.81)$ & Q & & $675(1.84)$ & Q \\
& $625(1.98)$ & Q & & $630(1.97)$ & Q \\
& $453(2.74)$ & Soret & & $453(2.74)$ & Soret \\
\hline S11 & & & D11 & $694(1.79)$ & BODIPY \\
& $669(1.85)$ & Q & & $633(1.96)$ & Q \\
& $620(2.00)$ & Q & & $596(2.08)$ & Q \\
& $449(2.76)$ & Soret & & $452(2.74)$ & Soret \\
\hline
\end{tabular}

We also investigated the nature of the bands of both fragments in the dyads in terms of electronic transitions between MOs, and the results are presented in Table S2. The band of isolated BODIPY is mainly a transition $\mathrm{H}-3 \rightarrow \mathrm{L}$ or $\mathrm{H}-2 \rightarrow \mathrm{L}$ and it is very similar in the dyads, where the MOs are located in that fragment. In the case of the oxasmaragdyrin fragment, the $\mathrm{Q}$ bands are described by the Gouterman orbitals, which are $\mathrm{H}-1 \rightarrow \mathrm{L}$ and $\mathrm{H} \rightarrow \mathrm{L}+1$ transitions and $\mathrm{H}-1 \rightarrow \mathrm{L}+1$ transitions. No transitions $\mathrm{H} \rightarrow \mathrm{L}$ are found for $\mathrm{Q}$ bands in the dyads. Other molecular orbitals such as $\mathrm{H}-2, \mathrm{~L}+2$, and $\mathrm{L}+3$ also represent a contribution. For the Soret band, we also found $\mathrm{H}-2 \rightarrow \mathrm{L}+1, \mathrm{H}-$ $1 \rightarrow \mathrm{L}+2, \mathrm{H}-1 \rightarrow \mathrm{L}+3$, and $\mathrm{H}-2 \rightarrow \mathrm{L}+2$ transitions. All the $\mathrm{Q}$ and Soret bands are oxasmaragdyrin $\rightarrow$ oxasmaragdyrin transitions.

A complete view of the electronic absorption spectra of the dyads D1-D6 is shown in Figure S3 and S4, separated by groups depending of the bridge. Figure S3 shows the systems with alkyl chain as bridge, while Figure S4 displays those systems containing rings besides an Acet moiety as bridge.

It is noteworthy the appearance in Figure S3 of a wide and intense band at longer wavelengths for dyads D3 (975 nm), D5 (1031 nm), and D6 (1095 nm), which correspond to separated charge excited states of the oxasmaragdyrin $\rightarrow$ BODIPY type. In the case of D10 and D11 in Figure S4, the more intense band at about $700 \mathrm{~nm}$ corresponds to BODIPY $\rightarrow$ BODIPY transitions. Charge transfer bands occur at about 990-1200 nm with small oscillator strengths. For comparison, we included D3 in this 
figure. It is important to mention that the analysis of the theoretical absorption spectrum of the dyad $D_{\text {ref }}$ shows charge transfer bands in the visible region with very low intensity.

In summary, we have shown that the dyads D3, D5, and D6 present better properties than $\mathrm{D}_{\text {ref }}$ to be used in dye-sensitized solar cells due to the notorious charge transfer they present.

In order to represent in a more complete manner the excited states, we computed the natural transition orbitals $(\mathrm{NTO})^{85}$ of the separated charge excited states of the dyads mentioned above (D3, D5, D6). Figure 2 shows NTOs for D6, and Figure S5 presents NTOs for D3 and D5.

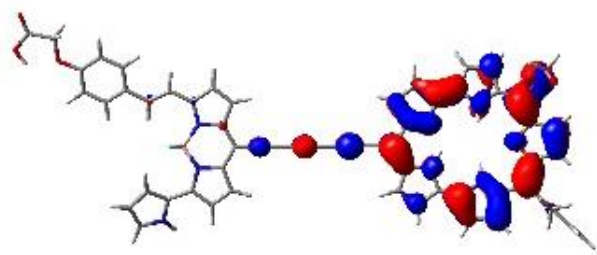

Occupied NTO

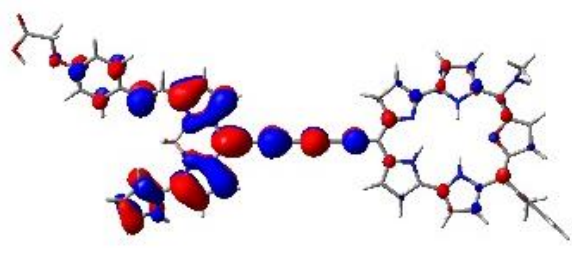

Unoccupied NTO

Figure 2. Surfaces of natural transition orbitals of the separate charge excited state of dyad D6 computed at the TPSSh/6-311G(d,p) level of theory in the solution phase $\left(\mathrm{CH}_{2} \mathrm{Cl}_{2}\right)$.

These dyads (D3, D5, D6) show an advantage with respect to other ones, in the sense that they absorb in a wider range of the electromagnetic spectrum, achieving a panchromatic absorption and an improvement for being used as photosensitizers in DSSC. The acetylene moiety favors such a panchromatic absorption. The acene groups (D10 and D11) are comparatively less favorable to charge transfer, but they present an increase in the intensity of the BODIPY absorption band (694-697 nm) in the dyad.

3.3 Aromatic Properties. The aromaticity of the molecular systems investigated in this work was analyzed on the basis of NICS and magnetically induced density current calculations. All the species show a ring critical point (RCP) of the electron density inside the macrocycle of the oxasmaragdyrin fragment. The electron density 
obtained at such an RCP is small and very similar for all the species studied, as the

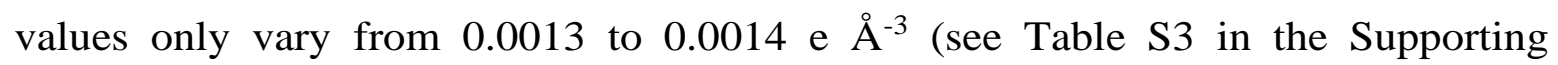
Information).

Table 5. NICS(0), bond distances between $\mathrm{C} 17$ and the linker $\mathrm{C}$ atom bonded to it, HOMOLUMO energy gap $\left(\Delta \mathrm{E}_{\mathrm{H} \rightarrow \mathrm{L}}\right)$, and the difference in energy between the LUMO $\left(\mathrm{L}^{\prime}\right)$ of $\mathrm{TiO}_{2}$ $(-4.3 \mathrm{eV})$ and the LUMO $(\mathrm{L})$ of $\mathrm{S} 1-\mathrm{S} 11$ and $\mathrm{D} 1-\mathrm{D} 11$ derivatives $\left(\Delta \mathrm{E}^{\prime}{ }_{\left.\mathrm{L} \rightarrow \mathrm{L}^{\prime}\right)}\right)$ calculated at the B3LYP/6-311G(d,p) level of theory.

\begin{tabular}{ccccc}
\hline Species & NICS $(0) / p p m$ & $\mathrm{~d}\left(\mathrm{C} 17-\mathrm{C}_{\text {linker }}\right) / \AA$ & $\Delta \mathrm{E}_{\mathrm{H} \rightarrow \mathrm{L}} / \mathrm{eV}$ & $\Delta \mathrm{E}^{\prime}{ }_{\mathrm{L} \rightarrow \mathrm{L}} / \mathrm{eV}$ \\
\hline S1 & -10.7 & & 2.40 & -2.28 \\
S2 & -11.2 & 1.509 & 2.46 & -2.30 \\
S3 & -12.1 & 1.421 & 2.28 & -2.01 \\
S4 & -11.8 & 1.420 & 2.30 & -2.13 \\
S5 & -12.3 & 1.411 & 2.15 & -1.83 \\
S6 & -12.4 & 1.406 & 2.06 & -1.68 \\
S7 & -11.6 & 1.482 & 2.38 & -2.13 \\
S8 & -11.8 & 1.413 & 2.16 & -1.95 \\
S9 & -11.7 & 1.481 & 2.39 & -2.15 \\
S10 & -11.6 & 1.492 & 2.43 & -2.16 \\
S11 & -11.6 & 1.486 & 2.41 & -2.14 \\
D1 & -12.0 & 1.487 & 1.87 & -1.48 \\
D2 & -11.2 & 1.511 & 1.70 & -1.44 \\
D3 & -12.1 & 1.405 & 1.68 & -1.17 \\
D4 & -12.0 & 1.419 & 1.64 & -1.38 \\
D5 & -12.3 & 1.403 & 1.62 & -1.11 \\
D6 & -12.3 & 1.402 & 1.56 & -1.04 \\
D7 & -11.6 & 1.482 & 1.54 & -1.18 \\
D8 & -12.0 & 1.411 & 1.71 & -1.35 \\
D9 & -11.4 & 1.484 & 1.44 & -1.14 \\
D10 & -11.5 & 1.493 & 1.54 & -1.16 \\
D11 & -11.4 & 1.485 & 1.44 & -1.12 \\
\hline
\end{tabular}

The aromaticity index NICS(0) calculated at RCP in S1-S11 species varies between -10.7 and $-12.4 \mathrm{ppm}$, thus indicating the aromatic character of the oxasmaragdyrin macrocycle in all these systems. These values are comparable to the $-16.5 \mathrm{ppm}$ value predicted for porphyrins ${ }^{86}$ and to the $-16.2,-14.9$ and $-14.1 \mathrm{ppm}$ values predicted for protonated pentaphyrins, sapphyrins, and isosmaragdyrins, respectively. ${ }^{87-88}$ As seen in Table 5, taking as reference S1, any group anchored to the C17 atom of this 
macrocycle (see Scheme 3 for atom numbering) gives rise to a slight increase in the aromaticity of the S1 moiety. For S2-S11 with respect to S1, the greatest increase in the aromaticity was found for S4 (1.1 ppm), S8 (1.1 ppm), S3 (1.4 ppm), S5 (1.6 ppm) and S6 (1.7 ppm). All these species have in common the presence of at least one triple $\mathrm{C} \equiv \mathrm{C}$ bond attached to $\mathrm{C} 17$, and the greater the number of the alternate $\mathrm{C} \equiv \mathrm{C}$ triple bonds, the higher the absolute value of $\operatorname{NICS}(0)$, and, so the aromaticity.

On the other hand, S7 and S9-S11, with an aromatic ring directly linked to C17, show a moderate rise of the aromaticity index of about $0.9-1.0 \mathrm{ppm}$. The number and type of the aryl ring do not seem to have a significant influence on the oxasmaragdyrin aromaticity. Finally, the smallest rise in the NICS(0) index was found for S2 (0.5 $\mathrm{ppm})$. NICS(0) values vary from -11.2 to $-12.3 \mathrm{ppm}$. When comparing NICS(0) values for S1-S11 with their corresponding dyads, D1-D11, hardly any change is observed ($0.1 \mathrm{ppm})-(0.2 \mathrm{ppm})$, except when oxasmaragdyrin is directly linked to BODIPY (1.3 ppm). The presence of the BODIPY moiety does not practically affect the aromaticity of the macrocycle and the trends found for S2-S11, unless when it directly attaches S1.

To get a broader knowledge of the aromaticity of the oxasmaragdyrin fragment in this kind of molecular systems, we also carried out magnetically induced current density calculations. To visualize the motion of electrons in such systems, streamline plots of the current densities calculated in a plane placed $1 \AA$ above the molecular plane and current pathways obtained by numerical integration of the current densities passing chemical bonds are shown in Figure 3 (see also Figure S6).

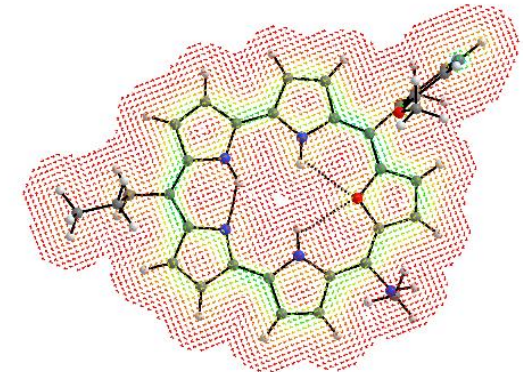

S2

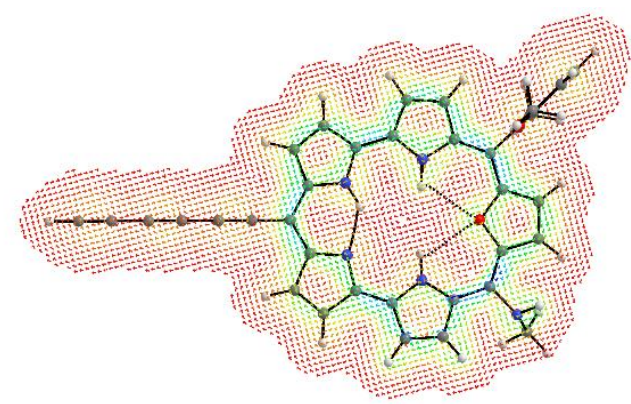

S6 
Figure 3. Current density trajectories which intersect a plane $1.0 \AA$ above the nuclear plane of the oxasmaragdyrin macrocycle for S2 and S6 and induced by a magnetic field applied along the $+Z$ axis, i.e. coming out of the page. Arrows indicate the direction of current flow. Arrows in blue are associated with higher current densities while arrows in red are ascribed to lower current densities. Counterclockwise flow is paramagnetic whereas clockwise flow is diamagnetic.

To obtain reliable current density maps, the greatest disadvantage comes from the deviation of the planarity of the oxasmaragdyrin macrocycle. Table 1 shows RMSD of the non-hydrogen atoms of the oxasmaragdyrin macrocycle to the best plane obtained fitting with the Cartesian coordinates of such atoms by the least squares method. A greater value of RMSD means lower planarity of the oxasmaragdyrin macrocycle. For such kind of non-planar species, it is difficult to define accurate current density maps. Under these circumstances, we focus on the species showing the largest aromatic index for the oxasmaragdyrin macrocycle, i.e., S6, and on the species displaying the least aromatic index for the macrocycle, i.e., S2 (see density maps for the remaining oxasmaragdyrin compounds in Figure S6). As can be seen in Figure 3, the current density flows through all the bonds implying the non-hydrogen atoms of the macrocycle of S6 in the clockwise direction, thus indicating the diatropic character of the ring current. Following the clockwise direction, the current density splits at each pyrrole ring into an inner $\mathrm{C}-\mathrm{N} / \mathrm{O}$ and an outer all carbon route. The outer route is preferred over the inner one. The presence of an alkyl group bonded to $\mathrm{C} 17$ in $\mathrm{S} 2$, decreases the current density flowing along the oxasmaragdyrin macrocycle, in agreement with the smaller NICS(0) index compared to that found in the case of S6, where the current density splits at each ring, with the outer route preferred over the inner one for all the rings except R4 (see Scheme 3 for ring numbering).

Trying to find an explanation for the above-mentioned trends, we investigated the relationship of the oxasmaragdyrin aromaticity with the planarity of such a macrocycle, the HOMO-LUMO energy gap, and the charge transport (see Tables 1, 5 and S4) of the whole set of molecular systems. In general, apart from S1, there is an anti-correlation between aromaticity and planarity. The smallest degree of planarity of the macrocycle corresponds to $\mathrm{S} 1$, which has the lowest absolute value for NICS(0), that is, it is the least aromatic compound. S3-S6 and S8 are also far from planarity 
(RMSD around $0.12 \AA$ ), although they show the largest aromaticity (the absolute value of $\operatorname{NICS}(0)$ is greater than or equal to $11.7 \mathrm{ppm})$. The presence of alternating $\mathrm{C} \equiv \mathrm{C}$ triple bonds seems to be better for the electron delocalization from the macrocycle to the bridge. Looking at Table 5, these most aromatic systems, S3-S6 and S8, present the shortest distances between $\mathrm{C} 17$ in the oxasmaragdyrin and the bridge (1.421-1.406 $\AA$ and 1.405-1.402 A, respectively), thus confirming a better electron delocalization. The greatest degree of planarity was found for S10 (RMSD = $0.06 \AA$ ) and S9 (RMSD $=0.05 \AA)$, which present moderate negative $\operatorname{NICS}(0)$ values around $-11.6 \mathrm{ppm}$, indicative of low aromaticity. In these systems the distance between $\mathrm{C} 17$ and the bridge is large (1.481 $\AA$ for S9 and $1.492 \AA$ for S10), thus indicating no delocalization of the electron density towards the linker. System S2 behaves similarly to the oxasmaragdyrins, with an aromatic ring directly linked to S1. It should be noted that dyads show a slight loss of planarity compared to their respective oxasmaragdyrin fragments, except for D1, D5, and D6. The relationships among planarity, aromaticity, and C17-C $\mathrm{C}_{\text {bridge }}$ bond distance found for S2-S11 also hold for D2-D11.

Concerning the HOMO-LUMO energy gap, for the isolated oxasmaragdyrins the smallest values were found for S6 (2.06 eV), S5 (2.15 eV), and S8 (2.16 eV), and the largest for S11 (2.41 eV) and S10 (2.43 eV). This trend is reversed when analyzing the HOMO-LUMO energy gaps for the corresponding dyads, as D3-D6 show here larger energy gaps than D10 and D11. It should be noted that when going from S1S11 to the corresponding D1-D11, the HOMO-LUMO energy gap decreases in the range of 0.47-0.97 eV (see Table 5), mainly due to the greater stabilization of the LUMO compared to that of the respective HOMO (see Table S4). We also found that all dyads studied here, at the exception of D1, D2, and D8, present smaller values for the HOMO-LUMO energy gap than $\mathrm{D}_{\text {ref }}(1.71 \mathrm{eV})$.

The HOMO-LUMO energy gaps obtained for S1-S11 are in the line of those obtained for porphyrin and other related systems. ${ }^{18,89-90}$ Smaller values were found for the D1-D11 dyads, and this trend is in principle good for DSSC applications. ${ }^{18}$ The LUMO energy level of S1-S11 and D1-D11 is always greater than the reported value of $-4.3 \mathrm{eV}$ for the edge level conduction band (CB) of $\mathrm{TiO}_{2}$ (LUMO') ) ${ }^{3,91-92}$ (negative values for the LUMO $\rightarrow$ LUMO' energy gap), thus favouring electron injection in the 
$\mathrm{CB}$ of $\mathrm{TiO}_{2}$. For S1-S11 and D1-D11, the most aromatic systems show small LUMO'LUMO energy gaps.

Indeed, S5 and S6 present energy gaps of -1.83 and $-1.68 \mathrm{eV}$ (the smallest in the absence of BODIPY), respectively, while values of -1.11 and $-1.04 \mathrm{eV}$ were found in the case of the corresponding dyads. We also note that D11 $(-1.12 \mathrm{eV})$ shows a value similar to that found for D5 and D6. In the case of $\mathrm{D}_{\text {ref, }}$ a similar trend is obtained with a value of $-1.21 \mathrm{eV}$.

For the recovery of the dye after the injection and charge transport, a mediator is needed and the dye's HOMO energy level has to be lower than the redox potential of the mediator $\left(\mathrm{E}_{\text {redox }}\right){ }^{18,89-93}$ For instance, in the case of the common $\mathrm{I}^{-} / \mathrm{I}_{3}{ }^{-}$mediator, its redox potential amounts to $-4.8 \mathrm{eV} .{ }^{93}$ Comparing the HOMO of S1-S11 and D1-D11 with -4.8 eV, D6 (-4.82 eV), D5 (-4.81 eV), and D3 (-4.80 eV), they are the only systems whose HOMO is slightly less than or equal to the reference value. Again, it seems there is a positive correlation between aromaticity and the $\mathrm{HOMO} \mathrm{dye}_{\mathrm{e}}-\mathrm{E}_{\mathrm{redox}}$ energy gap.

3.4 Charge Transport. Taking into account the charge transfer (CT) band shown in the absorption spectra, as an illustration, we calculated the electronic transport properties for two dyads; one with a significant CT band (D6) and the other without it (D4). Figure 4 shows the molecular junction system for D6 and the current direction as a function of the voltage sign (see arrows).

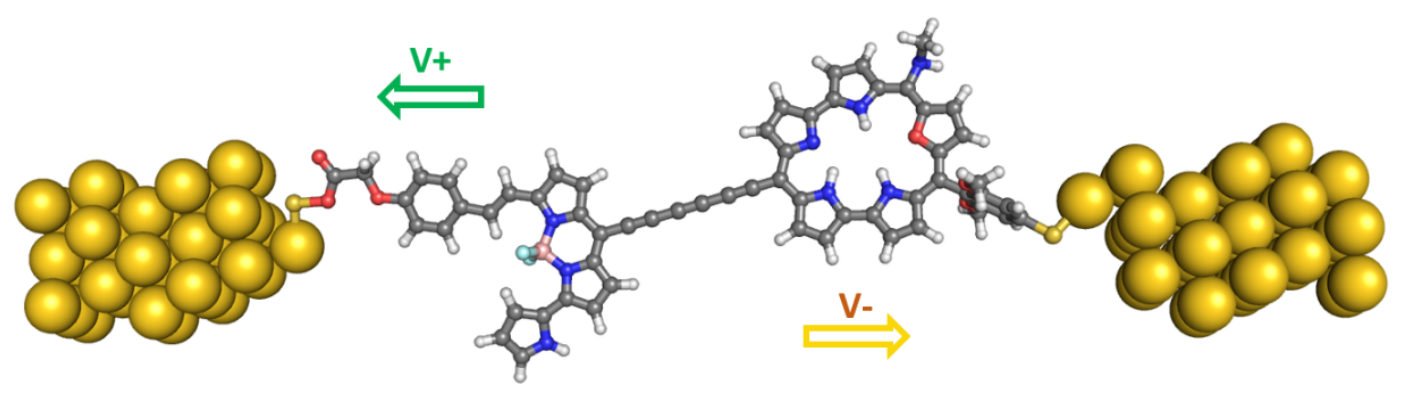


Figure 4. Scattering zone atomic structure for the studied molecular junction system composed of $\mathrm{Au}(111)-\mathrm{D} 6-\mathrm{Au}(111)$. Both electrodes (yellow) extend to infinity in the transport direction $\mathrm{z}$. The dyad is anchored to the electrode by one sulfur atom.

For the two studied systems, the conductance values obtained through DFT-NEGF methods were very small, with values around $10^{-12}$ and $10^{-9} \mathrm{G}_{0}$ for D4 and D6, respectively. However, despite the low conductance of both systems, a remarkable difference in conductivity is seen between them, with the conductance of the system presenting D6 as molecular bridge, around three orders of magnitude greater than that of its counterpart featuring D4, thus allowing us to expect that the electron transport is favored on the former. This trend could be confirmed by analyzing the energy-resolved transmission probabilities for both dyads shown in Figure 5 a for an energy range between $-1.0 \mathrm{eV}$ and $1.0 \mathrm{eV}$ with respect to the Fermi level, and in more detail from Figure $5 \mathrm{~b}$, where the transmission probabilities were depicted from -0.25 $\mathrm{eV}$ to $0.25 \mathrm{eV}$. It is seen that the system containing D6 has a significantly higher transmission probability in the proximities of the Fermi level, confirming the claim stated above.
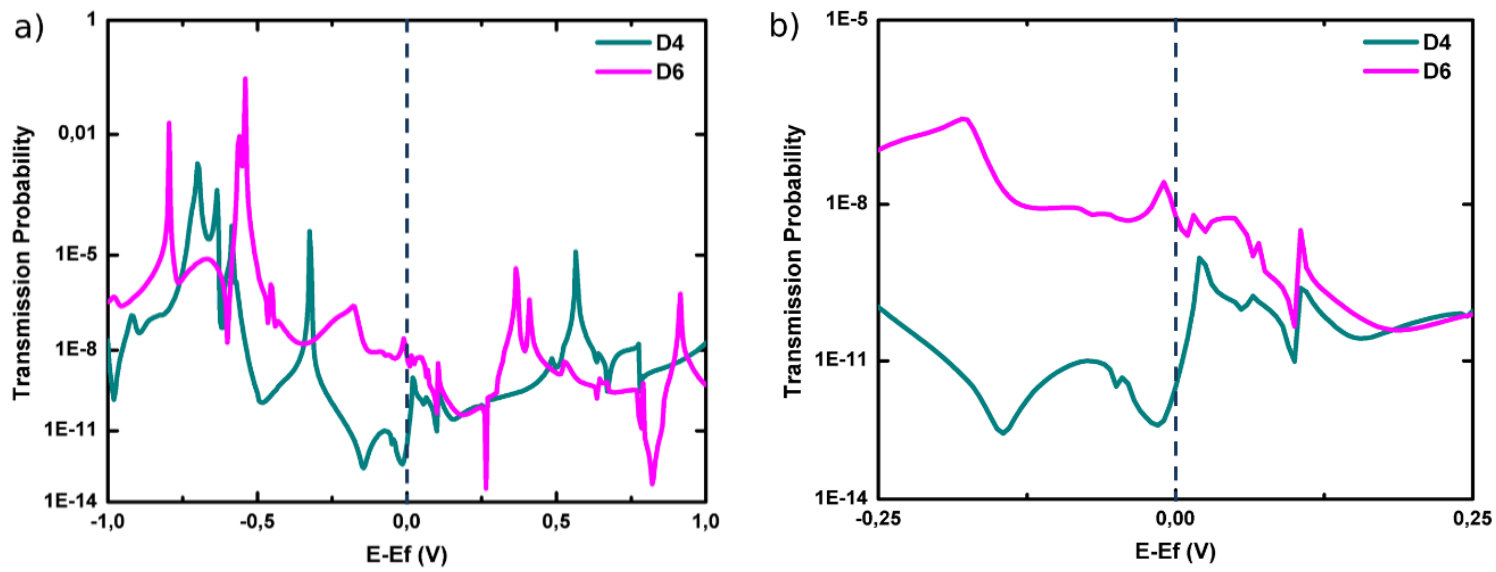

Figure 5. a) Transmission Probability for dyads D4 and D6, b) enlargement of the energy region closer to the Fermi level.

The current-voltage (I-V) profiles obtained for both systems for voltage ranges between -0.6 to $0.6 \mathrm{~V}$ and from -0.075 to $0.075 \mathrm{~V}$ are presented in Figure 6. 

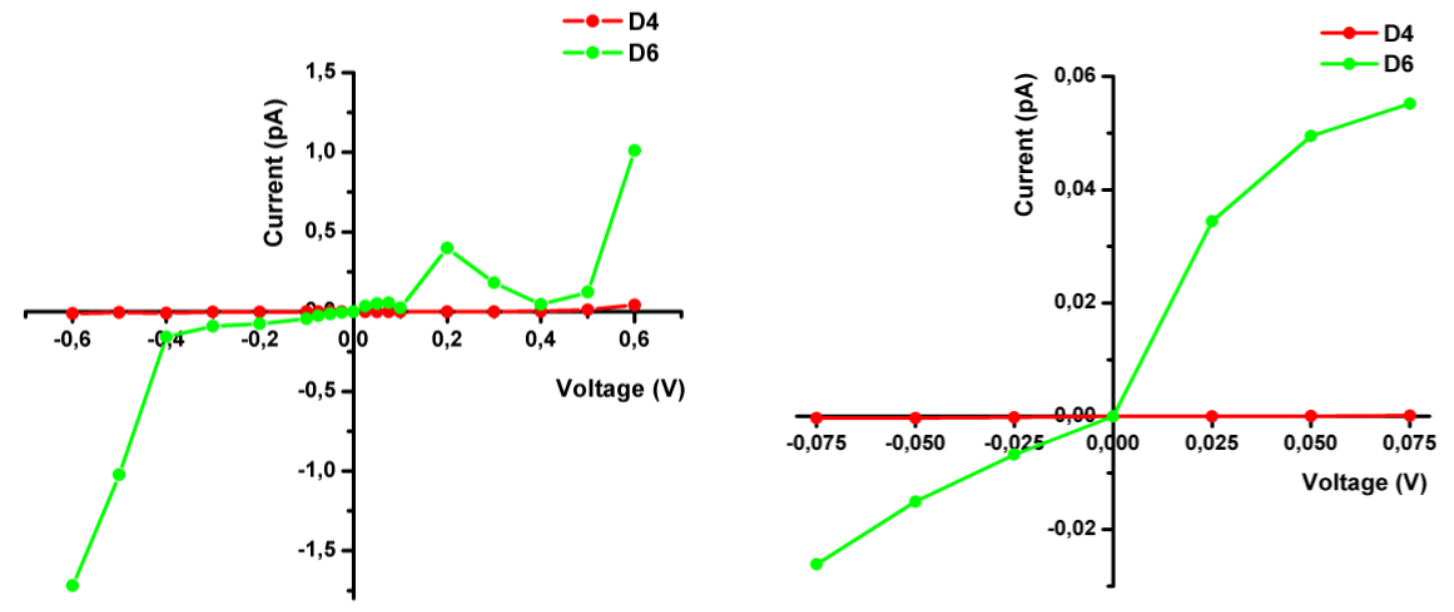

Figure 6. Current through Au111-D6(D4)-Au111 molecular device versus bias voltage.

Both systems exhibit small current values with strong differences between them for a given voltage, and for the system containing D6 the current is around two orders of magnitude greater for all the studied voltage range. This behavior is consistent with the values obtained above for the zero-bias conductance (Figure 6b), where the device composed of D6 shows a conductance of around three orders of magnitude greater than the D4 junction. Also, an asymmetric behavior is seen for both systems, with the direction of the current depending on the applied voltage sign. For positive voltage values, the current direction is from oxasmaragdyrin to BODIPY, and backward, if a negative bias voltage is applied. Our results confirm that the acetylene moieties favor the charge transport in the dyad.

3.5 Formation of the Dyads. To check if the synthesis of $D_{\text {ref }}$ reported by Kalita et al. ${ }^{37}$ (see Scheme 2) could be thermodynamically favorable for the synthesis of 11 dyads considered in this work, we calculated the Gibbs free energy change in toluene $\left(\Delta \mathrm{G}_{\text {solution}}\right)$ (see Table 6 ) for the reaction between the corresponding fragments, as well as the reference one for comparison purposes (see Scheme S2). For the sake of computational economy, we replaced I by $\mathrm{Br}$, since both are similar good leaving groups. 
Table 6. $\Delta \mathrm{G}_{\text {solution values }}(\mathrm{kcal} / \mathrm{mol})$ for the formation of the dyads calculated at the B3LYP/6-311G(d,p) level of theory in toluene. For comparison purpose, it is included the reference dyad.

\begin{tabular}{cccc}
\hline Dyad & $\Delta \mathrm{G}_{\text {solution }}$ & Dyad & $\Delta \mathrm{G}_{\text {solution }}$ \\
\hline${\text { D } \text { ref }^{\mathrm{a}}}^{\mathrm{N}}$ & -13.68 & $\mathrm{D} 6$ & -21.37 \\
D1 & -9.88 & $\mathrm{D} 7$ & -20.92 \\
D2 & -9.74 & $\mathrm{D} 8$ & -9.14 \\
D3 & -22.54 & $\mathrm{D} 9$ & -20.88 \\
D4 & -4.23 & $\mathrm{D} 10$ & -21.29 \\
D5 & -21.85 & D11 & -21.44 \\
\hline${ }^{\text {aRef }}{ }^{37}$ & & &
\end{tabular}

$\Delta \mathrm{G}_{\text {solution }}$ values are negative indicating that the formation of the dyads studied here is favorable. It means that all of them are stable and possible to be synthesized in the laboratory.

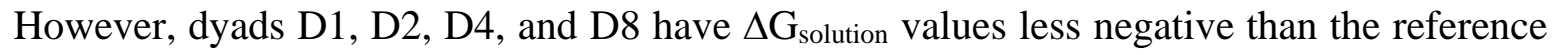
dyad $\left(D_{\text {ref }}\right)$. The remaining theoretical dyads produce more exergonic reactions than the experimental one, indicating that their stabilities are more favored.

The kind of bonding between BODIPY and oxasmaragdyrin clearly affects the stability of the dyad. Thus, bonding of BODIPY to $s p^{3}$ (like in D2 and D4) and $s p^{2}$ (like in D1 and D8) carbon atoms renders less stable dyads, whereas linking of BODIPY to an sp carbon atom (D3, D5, D6, D7, D9, D10, and D11) yields the most stable dyads. This seems to be a factor strongly affecting the exergonic character of the formation reaction of the dyads.

\section{CONCLUSIONS}

A density functional study of oxasmaragdyrin with 2,6-dimethoxyphenyl and methylamine groups in two of its meso carbons (S1), and the 4,4-difluoro-4-bora3a,4a-diaza-s-indacene (BODIPY, B) combined with alkyl, alkynyl, and aryl groups or combination of them forming dyads was carried out at the B3LYP/6-311G(d,p) and TPSSh/6-311G(d,p) levels of theory.

The study focused on the study of the influence of several bridging functional groups between B and S1 on a set of properties of the BODIPY-oxasmaragdyrin dyads: UV-vis electron absorption, aromaticity, and charge transport. 
Our results show that acetylene moieties as linkers between BODIPY and oxasmaragdyrin in the dyads favor the formation of separate charge excited states and also a panchromatic absorption.

On the other hand, the evaluation of $\operatorname{NICS}(0)$ and the magnetically induced current density for the oxasmaragdyrin fragment in the dyad and in its isolated form showed an agreement in the aromaticity. We found that the higher the aromaticity of the oxasmaragdyrin macrocycle, the greater the capacity for light-harvesting in a broader region of the electromagnetic spectrum. For all the oxasmaragdyrin fragments and the dyads, the LUMO'-LUMO energy gaps indicate that the electron injection toward $\mathrm{TiO}_{2}$ is favored and the $\mathrm{HOMO}_{\text {dye }}-\mathrm{E}_{\text {redox }}$ energy gaps are enough to regenerate the dye. In summary, the results suggest that the investigated dyads may be used in DSSCs.

Using NEGF calculations for the D4 and D6 dyads, we showed that the D6 system with three acetylene moieties is a better conductor than D4, in agreement with the charge transfer results obtained from the photophysical properties.

Finally, the exergonic character of the reactions for the formation of the studied dyads, following the Kalita's procedure, suggests that they could be synthesized.

\section{ASSOCIATED CONTENT}

\section{Supporting Information}

The Supporting Information is available free of charge on the ACS Publications website at DOI:

View of the optimized molecular structures, calculation of the photophysical parameters and assignment of bands, simulated electronic absorption spectra, view of the natural transition orbitals of dyads, calculations of the electron density $\rho(r)$ and laplacian of $\rho(\mathrm{r})$, graphics of current density trajectories, frontier molecular orbitals (HOMO, LUMO), and proposed reaction for the formation of BODIPY-oxasmaragdyrin dyads (DOC). 


\section{AUTHOR INFORMATION}

\section{Corresponding Author}

*Email: gloria.cardenas@usach.cl

*Email: rlopez@uniovi.es

\section{ORCID}

G. Cárdenas-Jirón: 0000-0003-4305-8088

R. López: 0000-0001-8899-705X

\section{Notes}

The authors declare no competing financial interest

\section{ACKNOWLEDGMENTS}

We thank the financial support of CONICYT/CHILE under FONDECYT Project 1171719 (G.C.-J.) and USACH/CHILE under DICYT 021841CJ Postdoctoral Project (N.M.-P., G.C.-J), Proyecto Fortalecimiento USACH USA1799_CG241216 (G.C.-J.) and DICYT-Universidad de Santiago de Chile. We also acknowledged CONICYTPCHA/Doctorado Nacional/2015-21150093 for Doctoral Fellowship of M.B.-M. and for financial support to perform a research stay at the Universidad de Oviedo (Spain) under the supervision of Professor Ramón López, where part of this work was carried out. Powered@NLHPC: This research was partially supported by the supercomputing infrastructure of the NLHPC (ECM-02) of the Universidad de Chile.

R.L., M.I.M., and D.A. also thank the Ministerio de Economía y Competitividad (Grant No. CTQ2015-70231-P) and Universidad de Oviedo (Grant No. PAPI-18-PF05) of Spain for their financial support.

\section{References}

1. Vlachopoulos, N.; Liska, P.; Augustynski, J.; Graetzel, M., Very efficient visible light energy harvesting and conversion by spectral sensitization of high surface area polycrystalline titanium dioxide films. J. Am. Chem. Soc. 1988, 110 (4), 1216-1220. 
2. O'Regan, B.; Grätzel, M., A low-cost, high-efficiency solar cell based on dyesensitized colloidal TiO2 films. Nature 1991, 353, 737.

3. Hagfeldt, A.; Graetzel, M., Light-Induced Redox Reactions in Nanocrystalline Systems. Chem. Rev. 1995, 95 (1), 49-68.

4. Kay, A.; Grätzel, M., Low cost photovoltaic modules based on dye sensitized nanocrystalline titanium dioxide and carbon powder. Sol. Energ. Mat. Sol. C. 1996, 44 (1), 99-117.

5. Kalyanasundaram, K.; Grätzel, M., Applications of functionalized transition metal complexes in photonic and optoelectronic devices. Coord. Chem. Rev. 1998, 177 (1), 347414.

6. Hagfeldt, A.; Grätzel, M., Molecular Photovoltaics. Acc. Chem. Res. 2000, 33 (5), 269-277.

7. $\quad$ Grätzel, M., Dye-sensitized solar cells. J. Photoch. Photobio. C 2003, 4 (2), 145 153.

8. Smestad, G.; Bignozzi, C.; Argazzi, R., Testing of dye sensitized TiO2 solar cells I: Experimental photocurrent output and conversion efficiencies. Sol. Energ. Mat. Sol. C. 1994, $32(3), 259-272$.

9. Hagfeldt, A.; Boschloo, G.; Sun, L.; Kloo, L.; Pettersson, H., Dye-Sensitized Solar Cells. Chem. Rev. 2010, 110 (11), 6595-6663.

10. Vougioukalakis, G. C.; Philippopoulos, A. I.; Stergiopoulos, T.; Falaras, P., Contributions to the development of ruthenium-based sensitizers for dye-sensitized solar cells. Coord. Chem. Rev. 2011, 255 (21), 2602-2621.

11. Narayan, M. R., Review: Dye sensitized solar cells based on natural photosensitizers. Renew. Sus. Energ. Rev. 2012, 16 (1), 208-215.

12. Gong, J.; Sumathy, K.; Qiao, Q.; Zhou, Z., Review on dye-sensitized solar cells (DSSCs): Advanced techniques and research trends. Renew. Sus. Energ. Rev. 2017, 68, 234246.

13. Richhariya, G.; Kumar, A.; Tekasakul, P.; Gupta, B., Natural dyes for dye sensitized solar cell: A review. Renew. Sus. Energ. Rev. 2017, 69, 705-718.

14. Kalyanasundaram, K.; Grätzel, M., Dye-Sensitized Solar Cells for Direct Conversion of Sunlight to Electricity. Material Matters 2009, 4, 88.

15. Odobel, F.; Blart, E.; Lagrée, M.; Villieras, M.; Boujtita, H.; El Murr, N.; Caramori, S.; Alberto Bignozzi, C., Porphyrin dyes for TiO2 sensitization. J. Mater. Chem. 2003, 13 (3), 502-510.

16. Campbell, W. M.; Burrell, A. K.; Officer, D. L.; Jolley, K. W., Porphyrins as light harvesters in the dye-sensitised TiO2 solar cell. Coord. Chem. Rev. 2004, 248 (13), 13631379.

17. Wang, Q.; Campbell, W. M.; Bonfantani, E. E.; Jolley, K. W.; Officer, D. L.; Walsh, P. J.; Gordon, K.; Humphry-Baker, R.; Nazeeruddin, M. K.; Grätzel, M., Efficient Light Harvesting by Using Green Zn-Porphyrin-Sensitized Nanocrystalline TiO2 Films. J. Phys. Chem. B 2005, 109 (32), 15397-15409.

18. Balanay, M. P.; Kim, D. H., DFT/TD-DFT molecular design of porphyrin analogues for use in dye-sensitized solar cells. Phys. Chem. Chem. Phys. 2008, 10 (33), 5121-5127.

19. Dong, H.; Zhou, X.; Jiang, C., Molecular design and theoretical investigation on novel porphyrin derivatives for dye-sensitized solar cells. Theor. Chem. Acc. 2012, 131 (2), 1102 . 
20. Xie, M.; Wang, J.; Xia, H.-Q.; Bai, F.-Q.; Jia, R.; Rim, J.-G.; Zhang, H.-X., Theoretical studies on the spectroscopic properties of porphyrin derivatives for dyesensitized solar cell application. RSC Adv. 2015, 5 (42), 33653-33665.

21. Tanaka, T.; Osuka, A., Chemistry of meso-Aryl-Substituted Expanded Porphyrins: Aromaticity and Molecular Twist. Chem. Rev. 2017, 117 (4), 2584-2640.

22. Chatterjee, T.; Srinivasan, A.; Ravikanth, M.; Chandrashekar, T. K., Smaragdyrins and Sapphyrins Analogues. Chem. Rev. 2017, 117 (4), 3329-3376.

23. Gobeze, H. B.; Kumar, S.; D'Souza, F.; Ravikanth, M., Strongly Coupled Oxasmaragdyrin-BF2 Chelated Dipyrrin Dyads: Syntheses, X-ray Structure, Ground- and Excited-State Charge-Transfer Interactions. Chem.-Eur. J. 2017, 23 (7), 1546-1556.

24. Mane, S. B.; Sutanto, A. A.; Cheng, C.-F.; Xie, M.-Y.; Chen, C.-I.; Leonardus, M.; Yeh, S.-C.; Beyene, B. B.; Diau, E. W.-G.; Chen, C.-T.; Hung, C.-H., Oxasmaragdyrins as New and Efficient Hole-Transporting Materials for High-Performance Perovskite Solar Cells. ACS Appl. Mater. Inter. 2017, 9 (37), 31950-31958.

25. Mane, S. B.; Hu, J.-Y.; Chang, Y.-C.; Luo, L.; Diau, E. W.-G.; Hung, C.-H., Novel expanded porphyrin sensitized solar cells using boryl oxasmaragdyrin as the sensitizer. Chem. Commun. 2013, 49 (61), 6882-6884.

26. Cárdenas-Jirón, G.; Borges-Martínez, M.; Sikorski, E.; Baruah, T., Excited States of Light-Harvesting Systems Based on Fullerene/Graphene Oxide and Porphyrin/Smaragdyrin. J. Phys. Chem. C 2017, 121 (9), 4859-4872.

27. Jiang, X.-D.; Guan, J.; Zhao, J.; Le Guennic, B.; Jacquemin, D.; Zhang, Z.; Chen, S.; Xiao, L., Synthesis, structure and photophysical properties of NIR aza-BODIPYs with F/N3/NH2 groups at 1,7-positions. Dyes Pigments 2017, 136, 619-626.

28. Azarias, C.; Russo, R.; Cupellini, L.; Mennucci, B.; Jacquemin, D., Modeling excitation energy transfer in multi-BODIPY architectures. Phys. Chem. Chem. Phys. 2017, 19 (9), 6443-6453.

29. Grabarz, A. M.; Jędrzejewska, B.; Skotnicka, A.; Murugan, N. A.; Patalas, F.; Bartkowiak, W.; Jacquemin, D.; Ośmiałowski, B., The impact of the heteroatom in a fivemembered ring on the photophysical properties of difluoroborates. Dyes Pigments 2019, 170, 107481 .

30. Alberto, M. E.; De Simone, B. C.; Russo, N.; Sicilia, E.; Toscano, M., Can BODIPY Dimers Act as Photosensitizers in Photodynamic Therapy? A Theoretical Prediction. AIP Conf. Proc. 2018, 6 (143).

31. De Simone, B. C.; Mazzone, G.; Pirillo, J.; Russo, N.; Sicilia, E., Halogen atom effect on the photophysical properties of substituted aza-BODIPY derivatives. Phys. Chem. Chem. Phys. 2017, 19 (3), 2530-2536.

32. Alberto, M. E.; De Simone, B. C.; Mazzone, G.; Quartarolo, A. D.; Russo, N., Theoretical determination of electronic spectra and intersystem spin-orbit coupling: The case of isoindole-bodipy dyes. J. Chem. Theor. Comput. 2014, 10 (9), 4006-4013.

33. Pareek, Y.; Ravikanth, M., Synthesis and studies of covalently linked BF2oxasmaragdyrin-BODIPY and BF2-oxasmaragdyrin-ferrocene dyads. J. Porphyr. Phthalocya. 2013, 17 (01n02), 157-164.

34. Lin, C.-Y.; Wang, Y.-C.; Hsu, S.-J.; Lo, C.-F.; Diau, E. W.-G., Preparation and Spectral, Electrochemical, and Photovoltaic Properties of Acene-Modified Zinc Porphyrins. J. Phys. Chem. C 2010, 114 (1), 687-693. 
35. Liu, Y.; Xiang, N.; Feng, X.; Shen, P.; Zhou, W.; Weng, C.; Zhao, B.; Tan, S., Thiophene-linked porphyrin derivatives for dye-sensitized solar cells. Chem. Commun. 2009, (18), 2499-2501.

36. Lin, C.-Y.; Lo, C.-F.; Luo, L.; Lu, H.-P.; Hung, C.-S.; Diau, E. W.-G., Design and Characterization of Novel Porphyrins with Oligo(phenylethylnyl) Links of Varied Length for Dye-Sensitized Solar Cells: Synthesis and Optical, Electrochemical, and Photovoltaic Investigation. J. Phys. Chem. C 2009, 113 (2), 755-764.

37. Kalita, H.; Lee, W.-Z.; Ravikanth, M., Phosphorus Complexes of meso-Triaryl-25oxasmaragdyrins. Inorg. Chem. 2014, 53 (17), 9431-9438.

38. Urbani, M.; Grätzel, M.; Nazeeruddin, M. K.; Torres, T., Meso-Substituted Porphyrins for Dye-Sensitized Solar Cells. Chem. Rev. 2014, 114 (24), 12330-12396.

39. ThermoFisher https://www.thermofisher.com/cl/es/home/references/molecularprobes-the-handbook/fluorophores-and-their-amine-reactive-derivatives/bodipy-dyeseries.html.

40. Becke, A. D., Density-functional exchange-energy approximation with correct asymptotic behavior. Phys. Rev. A 1988, 38 (6), 3098-3100.

41. Lee, C.; Yang, W.; Parr, R. G., Development of the Colle-Salvetti correlation-energy formula into a functional of the electron density. Phys. Rev. B 1988, 37 (2), 785-789.

42. Becke, A. D., Density-functional thermochemistry. III. The role of exact exchange. J. Chem. Phys. 1993, 98 (7), 5648-5652.

43. Frisch, M. J.; Trucks, G. W.; Schlegel, H. B.; Scuseria, G. E.; Robb, M. A.; Cheeseman, J. R.; Scalmani, G.; Barone, V.; Mennucci, B.; Petersson, G. A. et al. Gaussian 092009.

44. Hehre, W. J.; Radom, L.; Pople, J. A.; Schleyer, P. v. R., Ab Initio Molecular Orbital Theory; Wiley: New York, 1986.

45. Schlegel, H. B., Optimization of equilibrium geometries and transition structures. $J$. Comput. Chem. 1982, 3 (2), 214-218.

46. Bernhard Schlegel, H., Estimating the hessian for gradient-type geometry optimizations. Theor. Chim. Acta 1984, 66 (5), 333-340.

47. Li, X.; Frisch, M. J., Energy-Represented Direct Inversion in the Iterative Subspace within a Hybrid Geometry Optimization Method. J. Chem. Theor. Comput. 2006, 2 (3), 835839 .

48. Grimme, S.; Antony, J.; Ehrlich, S.; Krieg, H., A consistent and accurate ab initio parametrization of density functional dispersion correction (DFT-D) for the 94 elements HPu. J. Chem. Phys. 2010, 132 (15), 154104.

49. Furche, F.; Ahlrichs, R., Adiabatic time-dependent density functional methods for excited state properties. J. Chem. Phys. 2002, 117 (16), 7433-7447.

50. Stratmann, R. E.; Scuseria, G. E.; Frisch, M. J., An efficient implementation of timedependent density-functional theory for the calculation of excitation energies of large molecules. J. Chem. Phys. 1998, 109 (19), 8218-8224.

51. Bauernschmitt, R.; Ahlrichs, R., Treatment of electronic excitations within the adiabatic approximation of time dependent density functional theory. Chem. Phys. Lett. 1996, 256 (4), 454-464.

52. Sundholm, D., Interpretation of the electronic absorption spectrum of free-base porphin using time-dependent density-functional theory. Phys. Chem. Chem. Phys. 2000, 2 (10), 2275-2281. 
53. Becke, A. D., A new mixing of Hartree-Fock and local density-functional theories. J. Chem. Phys. 1993, 98 (2), 1372-1377.

54. Yanai, T.; Tew, D. P.; Handy, N. C., A new hybrid exchange-correlation functional using the Coulomb-attenuating method (CAM-B3LYP). Chem. Phys. Lett. 2004, 393 (1), 5157.

55. Adamo, C.; Barone, V., Toward reliable density functional methods without adjustable parameters: The PBE0 model. J. Chem. Phys. 1999, 110 (13), 6158-6170.

56. Tao, J.; Perdew, J. P.; Staroverov, V. N.; Scuseria, G. E., Climbing the Density Functional Ladder: Nonempirical Meta--Generalized Gradient Approximation Designed for Molecules and Solids. Phys. Rev. Lett. 2003, 91 (14), 146401.

57. Staroverov, V. N.; Scuseria, G. E.; Tao, J.; Perdew, J. P., Comparative assessment of a new nonempirical density functional: Molecules and hydrogen-bonded complexes. $J$. Chem. Phys. 2003, 119 (23), 12129-12137.

58. Chai, J.-D.; Head-Gordon, M., Systematic optimization of long-range corrected hybrid density functionals. J. Chem. Phys. 2008, 128 (8), 084106.

59. Narayanan, S. J.; Sridevi, B.; Chandrashekar, T. K.; Englich, U.; Ruhlandt-Senge, K., Core-Modified Smaragdyrins: First Examples of Stable Meso-Substituted Expanded Corrole. Org. Lett. 1999, 1 (4), 587-590.

60. Barone, V.; Cossi, M., Quantum Calculation of Molecular Energies and Energy Gradients in Solution by a Conductor Solvent Model. J. Phys. Chem. A 1998, 102 (11), 19952001.

61. Cossi, M.; Rega, N.; Scalmani, G.; Barone, V., Energies, structures, and electronic properties of molecules in solution with the C-PCM solvation model. J. Comput. Chem. 2003, 24 (6), 669-681.

62. Schleyer, P. v. R.; Maerker, C.; Dransfeld, A.; Jiao, H.; van Eikema Hommes, N. J. R., Nucleus-Independent Chemical Shifts: A Simple and Efficient Aromaticity Probe. J. Am. Chem. Soc. 1996, 118 (26), 6317-6318.

63. Chen, Z.; Wannere, C. S.; Corminboeuf, C.; Puchta, R.; Schleyer, P. v. R., NucleusIndependent Chemical Shifts (NICS) as an Aromaticity Criterion. Chem. Rev. 2005, 105 (10), 3842-3888.

64. Morao, I.; Lecea, B.; Cossío, F. P., In-Plane Aromaticity in 1,3-Dipolar Cycloadditions. J. Org. Chem. 1997, 62 (20), 7033-7036.

65. Bader, R. F. W., Atoms in Molecules. A Quantum Theory. University Press: Oxford, 1990.

66. Bader, R. F. W.; Popelier, P. L. A.; Keith, T. A., Theoretical Definition of a Functional Group and the Molecular Orbital Paradigm. Angew. Chem. Int. Ed. 1994, 33 (6), 620-631.

67. Popelier, P. L., Atoms in Molecules: An Introduction. Prentice Hall: London, 2000.

68. Keith, T. A.; T. K. Gristmill Software, Overland Park KS, AIMAll (Version 11.05.16), 2011. http://aim.tkgristmill.com/

69. Schleyer, P. v. R.; Jiao, H.; Hommes, N. J. R. v. E.; Malkin, V. G.; Malkina, O. L., An Evaluation of the Aromaticity of Inorganic Rings: Refined Evidence from Magnetic Properties. J. Am. Chem. Soc. 1997, 119 (51), 12669-12670.

70. Schleyer, P. v. R.; Manoharan, M.; Wang, Z.-X.; Kiran, B.; Jiao, H.; Puchta, R.; van Eikema Hommes, N. J. R., Dissected Nucleus-Independent Chemical Shift Analysis of $\pi$ Aromaticity and Antiaromaticity. Org. Lett. 2001, 3 (16), 2465-2468. 
71. Stuyver, T.; Perrin, M.; Geerlings, P.; De Proft, F.; Alonso, M., Conductance Switching in Expanded Porphyrins through Aromaticity and Topology Changes. J. Am. Chem. Soc. 2018, 140 (4), 1313-1326.

72. Keith, T. A.; Bader, R. F. W., Topological analysis of magnetically induced molecular current distributions. J. Chem. Phys. 1993, 99 (5), 3669-3682.

73. Keith, T. A., Calculation of magnetizabilities using GIAO current density distributions. Chem. Phys. 1996, 213 (1), 123-132.

74. Valiev, R. R.; Cherepanov, V. N., The influence of benzene rings on aromatic pathways in the porphyrins. Int. J. Quantum Chem. 2013, 113 (23), 2563-2567.

75. Valiev, R. R.; Fliegl, H.; Sundholm, D., Aromatic Pathways in Carbathiaporphyrins. J. Phys. Chem. A 2015, 119 (7), 1201-1207.

76. Franzke, Y. J.; Sundholm, D.; Weigend, F., Calculations of current densities and aromatic pathways in cyclic porphyrin and isoporphyrin arrays. Phys. Chem. Chem. Phys. 2017, 19 (20), 12794-12803.

77. Waldron, D.; Haney, P.; Larade, B.; MacDonald, A.; Guo, H., Nonlinear spin current and magnetoresistance of molecular tunnel junctions. Phys. Rev. Lett. 2006, 96 (16), 166804. 78. Taylor, J.; Guo, H.; Wang, J., Ab initio modeling of quantum transport properties of molecular electronic devices. Phys. Rev. B 2001, 63 (24), 245407.

79. Perdew, J. P., Erratum: Density-functional approximation for the correlation energy of the inhomogeneous electron gas. Phys. Rev. B 1986, 34 (10), 7406-7406.

80. Weigend, F.; Ahlrichs, R., Balanced basis sets of split valence, triple zeta valence and quadruple zeta valence quality for $\mathrm{H}$ to $\mathrm{Rn}$ : Design and assessment of accuracy. Phys. Chem. Chem. Phys. 2005, 7 (18), 3297-3305.

81. Rappoport, D.; Furche, F., Property-optimized Gaussian basis sets for molecular response calculations. J. Chem. Phys. 2010, 133 (13), 134105.

82. Ordejón, P.; Artacho, E.; Soler, J. M., Self-consistent order-\$N\$ density-functional calculations for very large systems. Phys. Rev. B 1996, 53 (16), R10441-R10444.

83. Soler, J. M.; Artacho, E.; Gale, J. D.; Garcia, A.; Junquera, J.; Ordejon, P.; SanchezPortal, D. The SIESTA method for ab initio order-N materials simulation arXiv e-prints [Online], 2001. https://ui.adsabs.harvard.edu/abs/2001cond.mat.11138S (accessed November 01, 2001).

84. Perdew, J. P.; Burke, K.; Ernzerhof, M., Generalized Gradient Approximation Made Simple. Phys. Rev. Lett. 1996, 77 (18), 3865-3868.

85. Martin, R. L., Natural transition orbitals. J. Chem. Phys. 2003, 118 (11), 4775-4777.

86. Cyrañski, M. K.; Krygowski, T. M.; Wisiorowski, M.; van Eikema Hommes, N. J. R.; Schleyer, P. v. R., Global and Local Aromaticity in Porphyrins: An Analysis Based on Molecular Geometries and Nucleus-Independent Chemical Shifts. Angew. Chem. Int. Edit. 1998, 37 (1-2), 177-180.

87. Yoon, Z. S.; Cho, D.-G.; Kim, K. S.; Sessler, J. L.; Kim, D., Nonlinear Optical Properties as a Guide to Aromaticity in Congeneric Pentapyrrolic Expanded Porphyrins: Pentaphyrin, Sapphyrin, Isosmaragdyrin, and Orangarin. J. Am. Chem. Soc. 2008, 130 (22), 6930-6931.

88. Mack, J., Expanded, Contracted, and Isomeric Porphyrins: Theoretical Aspects. Chem. Rev. 2017, 117 (4), 3444-3478.

89. Nazeeruddin, M. K.; De Angelis, F.; Fantacci, S.; Selloni, A.; Viscardi, G.; Liska, P.; Ito, S.; Takeru, B.; Grätzel, M., Combined Experimental and DFT-TDDFT Computational 
Study of Photoelectrochemical Cell Ruthenium Sensitizers. J. Am. Chem. Soc. 2005, 127 (48), 16835-16847.

90. Lu, X.; Wei, S.; Wu, C.-M. L.; Li, S.; Guo, W., Can Polypyridyl Cu(I)-based Complexes Provide Promising Sensitizers for Dye-Sensitized Solar Cells? A Theoretical Insight into $\mathrm{Cu}(\mathrm{I})$ versus Ru(II) Sensitizers. J. Phys. Chem. C 2011, 115 (9), 3753-3761.

91. Asbury, J. B.; Wang, Y.-Q.; Hao, E.; Ghosh, H. N.; Lian, T., Evidences of hot excited state electron injection from sensitizer molecules to $\mathrm{TiO} 2$ nanocrystalline thin films. Res. Chem. Intermediat. 2001, 27 (4), 393-406.

92. Mosurkal, R.; He, J.-A.; Yang, K.; Samuelson, L. A.; Kumar, J., Organic photosensitizers with catechol groups for dye-sensitized photovoltaics. J. Photoch. Photobio. A 2004, 168 (3), 191-196.

93. Cahen, D.; Hodes, G.; Grätzel, M.; Guillemoles, J. F.; Riess, I., Nature of Photovoltaic Action in Dye-Sensitized Solar Cells. J. Phys. Chem. B 2000, 104 (9), 20532059. 


\section{TOC}

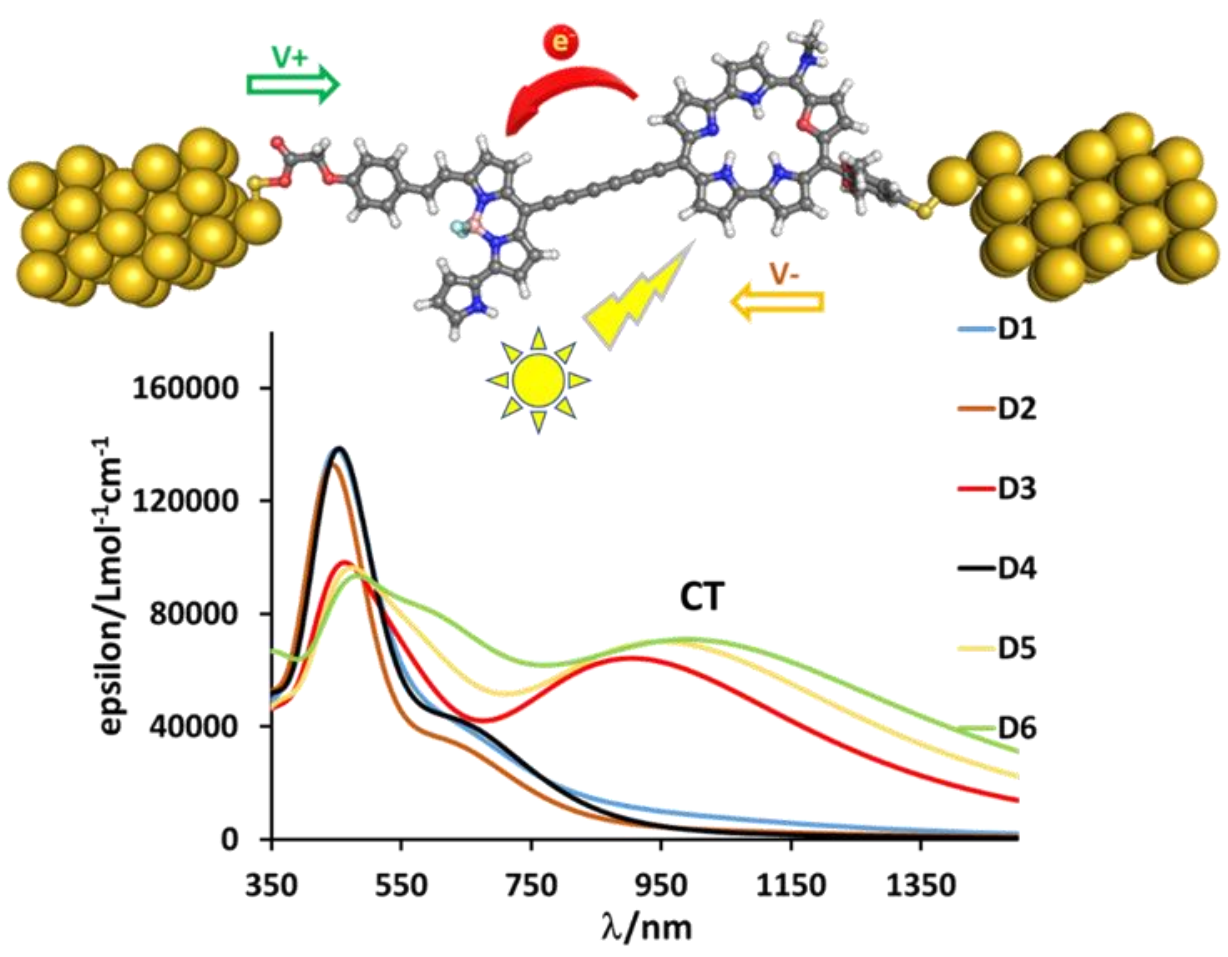

